\title{
Wnt5 and Drl/Ryk Gradients Pattern the Drosophila Olfactory Dendritic Map
}

\author{
Yuping Wu, ${ }^{1}$ Jay-Christian Helt, ${ }^{2}$ Emily Wexler, ${ }^{2}$ Iveta M. Petrova, ${ }^{3}$ Jasprina N. Noordermeer, ${ }^{3}$ Lee G. Fradkin, ${ }^{3}$ \\ and Huey Hing ${ }^{2}$ \\ ${ }^{1}$ Department of Cell and Developmental Biology, University of Illinois at Urbana-Champaign, Urbana, Illinois 61801, 2Department of Biology, The College at \\ Brockport, SUNY, Brockport, New York 14420, and '3Laboratory of Developmental Neurobiology, Department of Molecular Cell Biology, Leiden University \\ Medical Center, 2300 RC Leiden, The Netherlands
}

During development, dendrites migrate to their correct locations in response to environmental cues. The mechanisms of dendritic guidance are poorly understood. Recent work has shown that the Drosophila olfactory map is initially formed by the spatial segregation of the projection neuron (PN) dendrites in the developing antennal lobe (AL). We report here that between 16 and $30 \mathrm{~h}$ after puparium formation, the PN dendrites undergo dramatic rotational reordering to achieve their final glomerular positions. During this period, a novel set of AL-extrinsic neurons express high levels of the Wnt5 protein and are tightly associated with the dorsolateral edge of the AL. Wnt5 forms a dorsolateral-high to ventromedial-low pattern in the antennal lobe neuropil. Loss of Wnt5 prevents the ventral targeting of the dendrites, whereas Wnt5 overexpression disrupts dendritic patterning. We find that Drl/Ryk, a known Wnt5 receptor, is expressed in a dorsolateral-to-ventromedial (DL > VM) gradient by the PN dendrites. Loss of Drl in the PNs results in the aberrant ventromedial targeting of the dendrites, a defect that is suppressed by reduction in Wnt5 gene dosage. Conversely, overexpression of Drl in the PNs results in the dorsolateral targeting of their dendrites, an effect that requires Drl's cytoplasmic domain. We propose that Wnt 5 acts as a repulsive guidance cue for the PN dendrites, whereas Drl signaling in the dendrites inhibits Wnt5 signaling. In this way, the precise expression patterns of Wnt5 and Drl orient the PN dendrites allowing them to target their final glomerular positions.

Key words: dendrite; Drl; RYK; signaling; targeting; Wnt

\section{Introduction}

During development, neurons elaborate axons and dendrites, which establish precise connections to form neural circuits. Recent work has shown that dendrites are highly dynamic and responsive to environmental cues (McAllister, 2000; Wong and Ghosh, 2002; Mauss et al., 2009; Rosso and Inestrosa, 2013). Indeed, the proper targeting of dendrites is important for the development and function of the nervous system (Corty et al., 2009; Jan and Jan, 2010). Given the central role of dendritic targeting in neural circuit formation, defects in dendritic targeting are associated with profound neurological diseases (Kaufmann and Moser, 2000; Walsh et al., 2008). Despite the importance of dendritic targeting, its underlying molecular mechanisms have remained poorly understood.

\footnotetext{
Received July 1, 2014; revised Sept. 2, 2014; accepted Sept. 30, 2014.

Author contributions: Y.W., L.G.F., and H.H. designed research; Y.W., J.-C.H., E.W., I.M.P., L.G.F., and H.H. performed research; Y.W., J.N.N., L.G.F., and H.H. analyzed data; J.N.N., L.G.F., and H.H. wrote the paper.

This work was supported by NIH/NIDCD Grants DC010916-01 (H.H.) and the Nederlandse Organisatie voor Wetenschappelijk Onderzoek (TOP Grant 40-00812-98-10058) and the Hersenstichting (HS 2011(1)-46; to L.G.F. and J.N.N.). We thank L. Luo, and the Bloomington Stock Center for providing fly stocks, and J. M. Dura and D. Eberl for their generous gifts of the anti-DRL antibody and PPTGAL4 vector, respectively.

The authors declare no competing financial interests.

Correspondence should be addressed to Dr Huey Hing, Department of Biology, The College at Brockport, SUNY, 350 New Campus Drive, Brockport, NY 14420. E-mail: hhing@brockport.edu.

DOI:10.1523/JNEUROSCI.2676-14.2014

Copyright $\odot 2014$ the authors $\quad 0270-6474 / 14 / 3414961-12 \$ 15.00 / 0$
}

The well defined neural circuitry of the Drosophila antennal lobes (ALs) offers an elegant system to investigate the mechanisms of dendritic targeting in neural circuit formation. Dendrites of 50 classes of projection neurons (PNs) stereotypically target 50 unique glomeruli where they form synapses with the axons of 50 classes of olfactory receptor neurons (ORNs; Couto et al., 2005; Fishilevich and Vosshall, 2005). The specificity with which the PN dendrites target their glomeruli is determined by their lineage and birth order (Jefferis et al., 2001). It was proposed that unique combinations of transcription factors direct the expression of unique sets of cell-surface receptors, allowing dendritic growth cones to terminate in specific glomeruli (Komiyama et al., 2003; Komiyama and Luo, 2007).

What guidance receptors and cues direct the targeting of the 50 classes of PN dendrites? We previously identified the wnt5 gene, which encodes a member of the non-canonical Wnt family of morphogens (Cadigan and Nusse, 1997), as a powerful regulator of AL development (Yao et al., 2007). Here, we show that the Wnt5 protein is distributed in a DL-high to VM-low pattern in the developing AL between 0 and $30 \mathrm{~h}$ after puparium formation (hAPF), a critical period for PN dendritic targeting. We show that a novel set of AL-extrinsic neurons, located at the dorsolateral edge of the AL, express the Wnt5 protein. Loss of wnt5 function abolished the ventral migration of dendrites, while overexpression of $w n t 5$ strongly disrupts the patterning of the dendritic map. 
We also show that the derailed ( $d r l$ ) gene, encoding a member of the Ryk family of atypical receptor tyrosine kinases and a putative Wnt5 receptor (Yoshikawa et al., 2003; Fradkin et al., 2004, 2010), is expressed by the PN dendrites in a decreasing DL $>$ VM gradient. Interestingly, the loss of $d r l$ promoted the ventromedial migration of PN dendrites, a phenotype that was strongly suppressed by the removal of a copy of the wnt 5 gene. We propose that Wnt5 acts as a repulsive guidance cue for the PN dendrites and Drl acts in the dendrites to inhibit Wnt5 signaling. We also propose that the spatially restricted expression pattern of Wnt5 orients the movement of the PN dendrites, allowing PN dendrites expressing different levels of Drl to localize to their appropriate positions along the DL-VM axis.

\section{Materials and Methods}

Transgenes. The UAS- $d r l$ and UAS- $d r l^{\Delta \text { intra }}$ transgenes were previously described by Yao et al. (2007). To introduce the GAL4 ORF into the wnt5 locus, we used "ends-out" homologous recombination-dependent gene targeting (Rong and Golic, 2000; Gong and Golic, 2003) using a derivative of the $p W 25$ vector (K. Golic, unpublished data; obtained from the Drosophila Genomic Resource Center). The GAL4 ORF was obtained by PCR from the pPTGAL4 plasmid (Sharma et al., 2002; a kind gift from D. Eberl, University of Iowa, Iowa City, IA) and cloned between the BsiW1 and Asc1 sites. Subsequently, $3 \mathrm{~kb}$ fragments of genomic sequences upstream of the wnt5 translation start codon and downstream of the stop codon were generated by PCR and directionally cloned into the BsiW1 and Kpn1/Not1 sites, respectively. The upstream fragment and downstream fragment encompassed, respectively, sequence positions 18402523-18399092 and 18395558-18393438 of the X chromosome (AE014298.4). All fragments were confirmed by DNA sequencing. The plasmid was introduced into the Drosophila germ line by standard P-element transgenesis to generate the $p W 25-w n t 5$-Gal4 donor insertion lines.

Generation of the $w n+5^{\text {Gal4 }}$ knock-in allele. Mobilization of the $p W 25-$ wnt5-Gal4 insert from a third chromosome donor was performed as described previously (Larsson et al., 2004). From $\sim 13,900$ progenies of the $p W 25-w n t 5-G a l 4 / h s-F L P$ hs-Cre females, eight $w n t 5^{\text {Galt }}$ lines were initially identified by genetic tests. All eight lines show the stereotyped wnt5 AL loss-of-function phenotype (Yao et al., 2007). Amplification of the insertions' junctional sequences followed by restriction enzyme analyses confirmed that all were precise replacement of the wnt 5 gene with the Gal4 gene. Faithful retention of the wild-type wnt5 expression pattern by $w n t 5^{\text {Gal4 }}$ was confirmed in two ways. First, expression of UAS-wnt5 under the control of $w n t 5^{\text {Gal }}$ significantly rescued the AL morphology (data not shown). Second, expression of UAS-mCD8::GFP under the control of $w n t 5^{\text {Gal4 }}$, and staining with anti-Wnt5 and anti-CD8 antibodies showed colocalization of the Wnt5 and CD8 proteins (Fig. 1H).

Immunohistochemistry. Dissection, fixing and staining of adult or pupal brains were performed as previously described (Ang et al., 2003, 2006). For anti-Wnt5 staining, unfixed brains were directly stained with anti-Wnt5 antibody in PBS $\left(2.5 \mathrm{~h}\right.$ at $\left.4^{\circ} \mathrm{C}\right)$, wash with PBS with goat serum, followed by fixation in PLP $\left(1 \mathrm{~h}, 25^{\circ} \mathrm{C}\right)$. Subsequent steps are the same as below. Rabbit anti-DRL was a generous gift from J. M. Dura (Institute of Human Genetics, CNRS, Montpellier, France); mAb nc82 (1:20; Wagh et al., 2006) was obtained from the Iowa Antibody Bank; rabbit anti-GFP (1:100), and rat anti-mCD8 $\mathrm{mAb}$ (1:100) were obtained from Invitrogen and Caltag, respectively. Affinity-purified rabbit antiWnt5 (Fradkin et al., 2004) and rabbit anti-Drl (Moreau-Fauvarque et al., 1998) were both used at 1:100 dilutions. The secondary antibodies, FITC-conjugated goat anti-rabbit, Cy3-conjugated goat anti-mouse and FITC-conjugated goat anti-rat, were obtained from The Jackson Laboratory and used at 1:100 dilutions.

Quantification of Wnt5, drl, and N-cadherin staining. Eight randomly chosen 16 hAPF ALs, doubly stained for N-Cadherin and either Wnt5 or Drl were and imaged using a Zeiss 710 confocal microscope. The Wnt5, $\mathrm{Drl}$, and $\mathrm{N}$-Cadherin expression patterns were quantified in the optical plane at the level of the DLC cell nerve ( $\sim 6 \mu \mathrm{m}$ from the anterior edge).
A DL-VM axis ( $\sim 22 \mu \mathrm{m}$ long) was manually drawn starting at the DLC neuron termini and ending at the ventromedial edge of the AL and pixel intensities along the line were calculated. The pixel intensity of Wnt5, or $\mathrm{Drl}$, at each point was divided by that of $\mathrm{N}$-Cadherin to give the normalized Wnt5, or Drl, value at that point. The data were analyzed and plotted using the Prism statistical software.

Quantification of the distribution of GH146-Gal4-labeled dendrites in the wnt5 mutant and wild-type ALs. Adult wnt 5 mutant or wild-type ALs expressing UAS-mCD8::GFP under the control of GH146-Gal4 were doubly stained with nc82 and anti-CD8. Six ALs were randomly chosen and imaged using a Zeiss 510 confocal microscope. For each AL, the CD8 and nc82 immunofluorescence of 10 optical sections were captured. The CD8 immunofluorescence of each section was divided into four quadrants (DL, DM, VL, VM, with the boundary defined by the extend of the nc82 staining) and the intensity of each quadrant was quantified using $\mathrm{NIH}$ ImageJ software. The intensities of each quadrant across the 10 sections were summed to give the subtotal for a quadrant and the percentage of CD8 staining intensity for the quadrant with respect to the total CD8 staining was then calculated and tabulated. The graph of the percentage of CD8 in the various sectors of the AL was generated using the Prism statistical software.

Quantification of the migration of GH146-Gal4- and Mz19-Gal4labeled dendrites in the wnt 5 mutant and wnt5/+ control ALs between 16 and $30 \mathrm{hAPF}$. Climbing third-instar larval expressing UAS-mCD8::GFP under the control of either GH146-Gal4 or Mz19-Gal4 drivers were separated into males ( $w n t 5$ mutants) or females (wnt5/+ controls). The ALs were stained at the selected developmental stages and imaged to visualize the PN dendrites. To measure the dendritic positions, a section $(\sim 6 \mu \mathrm{m}$ from the anterior of the AL) was chosen where the characteristic dendritic arbors could be unambiguously identified. A line was drawn through the arbors and the angle that the line made against the sagittal midline was measured. For each genotype and developmental stage, the dendrites of 10-12 ALs were measured. The data were compiled and analyzed and the graph generated using the Prism statistical software.

\section{Results}

\section{Wnt5 is found in two domains in the developing antennal lobe}

We previously identified the wnt5 gene in a screen for genes involved in AL patterning (Zhang et al., 2006) and showed its involvement in patterning the glomerular map during the period when the AL is organized by the incoming ORN axons (Yao et al., 2007). To understand how wnt5 might regulate the development of the AL at earlier stages, we examined its expression pattern in the AL at $16 \mathrm{hAPF}$, a time when the PN dendrites are segregating by directed targeting (Jefferis et al., 2004). Examination of Wnt5 staining at different planes along the anterior-posterior (A-P) axis of the AL showed that Wnt5 was concentrated in two regions, an anterior dorsolateral domain $(0-6 \mu \mathrm{m}$ from anterior) and to a lesser degree, a posterior ventrolateral domain (4-9 $\mu \mathrm{m}$ from anterior; Fig. 1A1,A2). Double-staining of ALs expressing UAS-mCD8::GFP under the control of GH146-Gal4, which labeled a large subset of the PNs, showed that the $\mathrm{Wnt} 5$ protein was extensively associated with PN dendrites (data not shown). Labeling of the DA1, VA1d and DC3 dendrites with the Mz19-Gal4 driver showed that the $M z 19$ dendrites reside selectively within the dorsolateral Wnt5 domain (Fig. 1A1). We noticed that, in addition to being highly concentrated in the dorsolateral and ventrolateral regions of the AL, Wnt5 was found in lower concentrations throughout the AL neuropil, suggesting that the protein diffused from the regions of high concentration (Fig. 1A1,A2). The Wnt5 antibody did not stain brains from the $w n t 5^{400}$-null mutant, confirming its specificity for the Wnt5 protein (Fig. 1A3). The high level of Wnt5 expression in the nascent AL was consistent with a role for Wnt5 in AL development. 

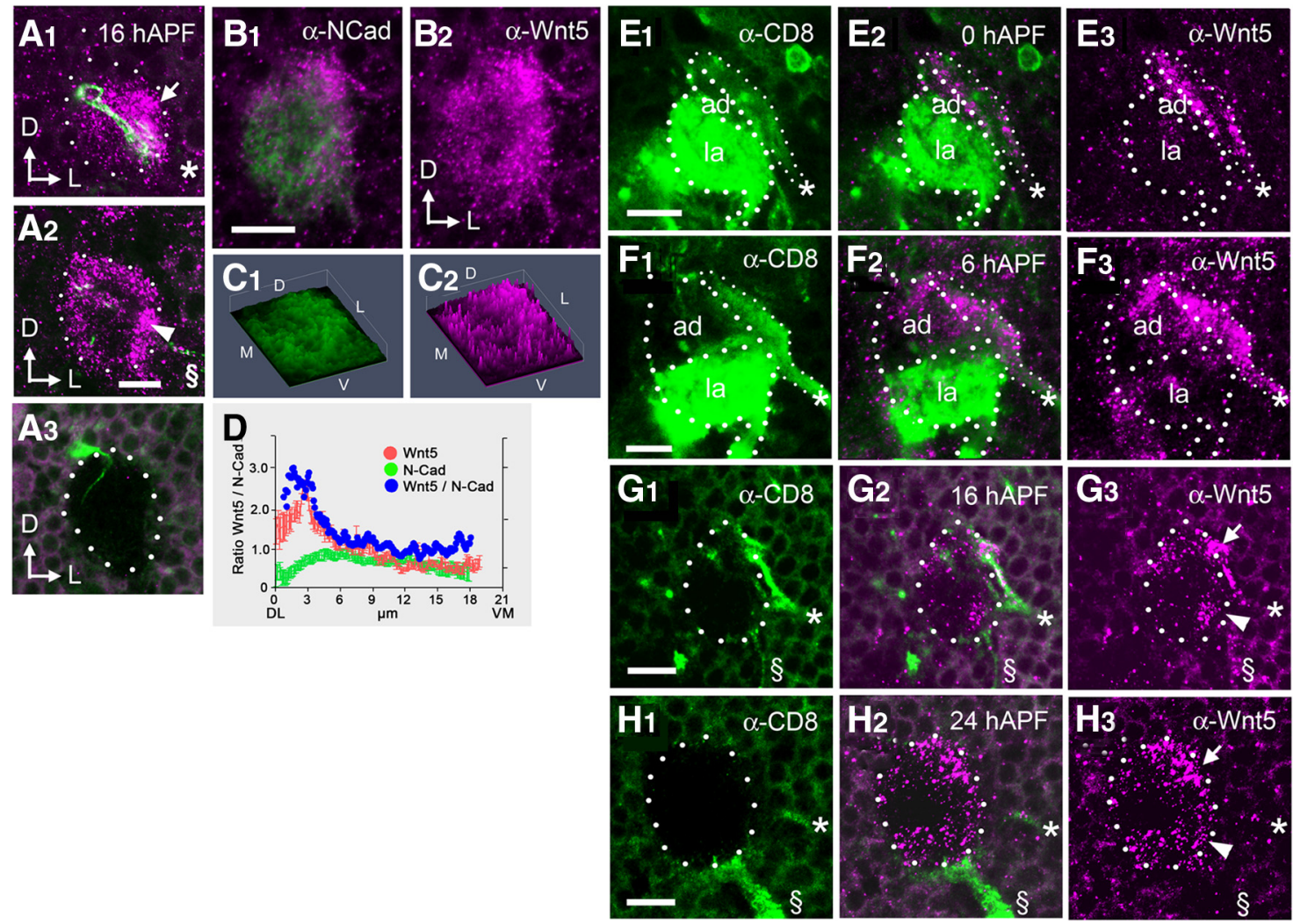

Figure 1. The Wnt5 and Drl proteins are expressed in decreasing dorsolateral-to-ventromedial gradients in the $16 \mathrm{hAPF}$ antennal lobe. Confocal sections of a $16 \mathrm{hAPF}$ AL from an Mz19-Gal4; UAS-mCD8::GFP animal, stained with antibodies against CD8 (green) and Wnt5 (magenta), at depths of $4 \mu \mathrm{m}(\boldsymbol{A 1})$ and $9 \mu \mathrm{m}(\boldsymbol{A 2})$, showing that the $M z 19$ dendrites are located in the anterior dorsolateral domain of Wnt5. № Wnt5 staining was observed in brains from the wnt $5^{400}$-null mutant, demonstrating the specificity of the Wnt5 antibody (A3). A representative wild-type 16 hAPF AL stained with antibodies against $\mathrm{N}$-Cadherin (green) and Wnt5 (magenta), showing that the Wnt5 protein is concentrated at the dorsolateral region of the AL (B1, B2). 3-D histogram plots of the pixel intensities of the N-Cadherin (green) and Wnt5 (magenta) proteins from B, showing that Wnt5 is enriched in the dorsolateral AL (C1, C2). A plot of the average N-Cadherin (green) and Wnt5 (red) staining intensities, as well as normalized Wnt5 values (blue) along the DL-VM axis of the AL, showing that Wnt5 is distributed in a DL-high versus VM-low pattern in the 16 hAPF AL. D, ALs are shown from wnt5 $5^{\text {Gal }} /+$; UAS-mCD8::GFP animals stained with antibodies against CD8 (green) and Wnt5 (magenta; $\boldsymbol{E}-\boldsymbol{H}$ ). Dotted lines highlight the boundaries of the ALs. At 0 hAPF, the developing adult $\mathrm{AL}(\mathrm{ad})$ was flanked by a thick bundle of GFP ${ }^{+}$fibers (the presumptive DLC neuronal processes, asterisk) along the dorsolateral edge, and the degenerating larval $\mathrm{AL}$ (la) along the ventromedial edge (E). The DLC neuronal fiber, but not the larval AL, is strongly stained by the Wnt5 antibody (E2, E3). A similar staining pattern expression is seen at $6 \mathrm{hAPF}(\boldsymbol{F})$. At $16 \mathrm{hAPF}$, the DLC nerve $\left(^{*}\right)$ was prominent while the larval AL had disappeared (G). A slender GFP ${ }^{+}$fiber, the antennal nerve (§), appeared at the ventrolateral edge of the AL, carrying adult ORN axons. The Wnt5 protein was found in two areas, a dorsolateral domain (arrow), associated with the DLC neuronal fiber, and a ventrolateral domain (arrowhead), associated with the antennal nerve (G2, G3). At 24 hAPF, the antennal nerve had grown thicker and ORN axons were beginning to encircle the AL $(\boldsymbol{H})$. The Wnt5 protein remained associated with the dorsolateral domain (arrow) and ventrolateral domain (arrowhead), although the DLC neuron fibers were now faintly visible (*). The expression pattern of Wnt5 was unchanged and remained fairly stable until $\sim 30 \mathrm{hAPF}$. Arrows in $\boldsymbol{A}$ and $\boldsymbol{B}$ indicate the dorsal and lateral axes. All ALs in this and subsequent figures are positioned dorsal side up and lateral side to the right. Scale bars, $10 \mu \mathrm{m}$.

\section{A novel cluster of neurons deposits Wnt5 in the dorsolateral antennal lobe}

Because Wnt5 is a secreted protein, anti-Wnt5 antibody staining has significant limitations in delineating the cells that express Wnt5. To identify the wnt5-expressing cells, we used genetargeting to precisely replace the wnt5 gene with the yeast Gal4 transcription factor gene (Gong and Golic, 2003) and created the $w n t 5^{\text {Gall }}$ knock-in allele. This allowed us to unambiguously visualize the cells that express $w n t 5$. The $w n t 5^{\text {Gal }}$ adult animals exhibited a strong wnt5 AL phenotype (Yao et al., 2007), which was rescued after the introduction of the UAS-wnt5 transgene (see Fig. $4 B, D$ ) suggesting that $w n t 5^{\text {Gal4 }}$ mimicked wnt5's wild-type expression pattern in the AL. We stained $w n t 5^{\text {Gal }} /+$ heterozygotes expressing UAS- $m C D 8:: G F P$ with Wnt5 and CD8 antibodies to visualize the wnt5-expressing cells. At 0 and $6 \mathrm{hAPF}$, we observed a thick $\mathrm{GFP}^{+}$fiber terminating on the dorsolateral edge of the nascent adult AL (Fig. 1E,F). This fiber was strongly stained by the Wnt 5 antibody. The degenerating larval AL, located on to the opposite, ventromedial edge, was also strongly labeled by GFP, but were not stained by the Wnt 5 antibody. At 16
hAPF, the larval AL had completely degenerated and the adult AL had grown larger (Fig. 1G). The $\mathrm{GFP}^{+}$fiber showed high level of Wnt5 staining and its terminal was clearly associated with the dorsolateral domain of Wnt5 in the AL (Fig. $1 G$ arrow). A second smaller $\mathrm{GFP}^{+}$fiber now appeared, touching the $\mathrm{AL}$ at the ventrolateral margin (Fig. 1G). The position of the fiber and its time of appearance suggested that it was the nascent antennal nerve, bearing the newly arriving adult ORN axons. The nascent antennal nerve was associated with the ventrolateral domain of Wnt5 expression (Fig. $1 G$, arrowhead). At $24 \mathrm{hAPF}$, the antennal nerve had grown thicker and ORN axons were observed to extend around the periphery of the AL (Fig. $1 H$ ). These protein colocalization experiments suggested that the dorsolateral fiber and, possibly, the ingrowing adult ORN axons were the sources of the Wnt5 protein in the AL neuropil. We deduce that Wnt5 diffuses throughout the AL neuropil from these locations. To quantify the pattern of Wnt5 distribution in the AL neuropil, we doublestained 16 hAPF ALs with antibodies against Wnt5 and $\mathrm{N}$-Cadherin (a control for neuropil density), and normalized the Wnt5 staining intensities over $\mathrm{N}$-Cadherin staining intensities 

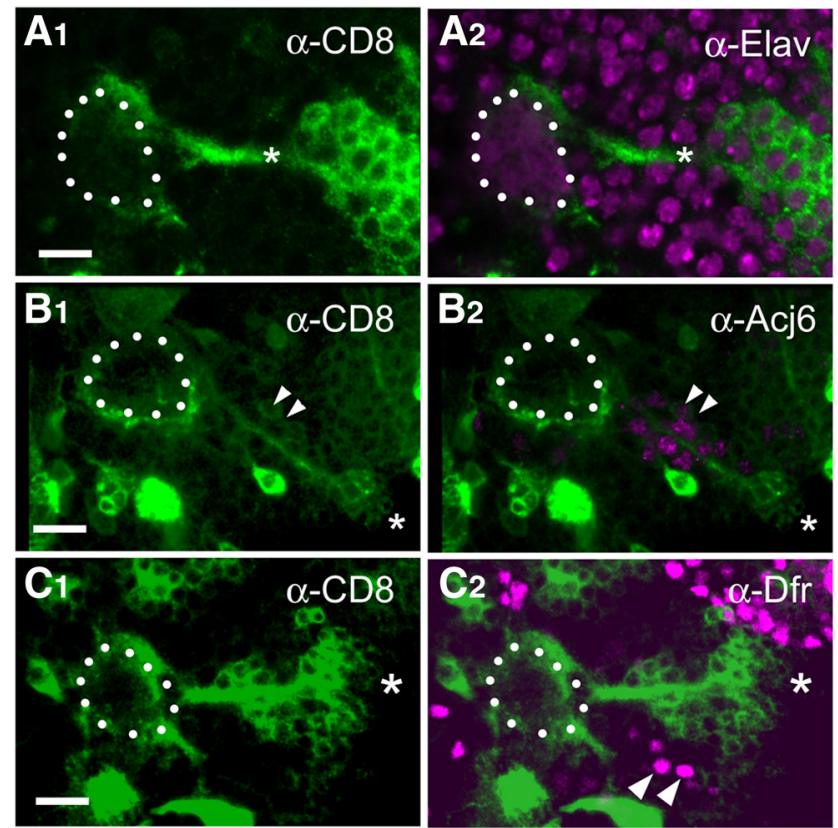

Figure 2. The Wnt5-expressing DLC cells are a previously undescribed type of AL-associated neurons. 16 hAPF ALs from wnt5 ${ }^{\text {Gal }}$; UAS-mCD8::GFP animals stained with antibodies against CD8 to visualize the wnt5-expressing cells (green) and against either Elav $(\boldsymbol{A}), \mathrm{Acj} 6(\boldsymbol{B})$, or Drifter (Dfr) (C; magenta). The developing $16 \mathrm{hAPF}$ AL is highlighted with dotted lines. The bundle of $\mathrm{GFP}^{+}$fibers terminating on the dorsolateral margin of the $\mathrm{AL}$ could be traced to a group of $\sim 20-30$ cells $\left({ }^{*}\right)$ located $\sim 50 \mu \mathrm{m}$ anterior and lateral to the AL in the deutocerebrum, which we called DLC cells (A1). The cells express the Elav protein in their nuclei indicating that they were neurons (A2). The cells did not express Acj6, or Dfr, although they are located close to clusters of Acj6- or Dfr-expressing PN cell bodies (B1, B2, C1, $\mathbf{C 2}$, arrowheads). These results, together with the observation that these cells did not extend dendrites into the AL or axons to the mushroom body suggested that they were not PNs, but were likely a novel type of ALassociated neurons. Scale bars, $10 \mu \mathrm{m}$.

(Fig. $1 B, C$ ). Plotting the normalized Wnt5 values along the DL-VM axis showed that Wnt5 forms a distinct pattern, being highly concentrated in the dorsolateral AL and declining to lower levels in central and ventromedial AL. (Fig. 1D).

We focused on characterizing the $\mathrm{Wnt} 5^{+}$cells terminating on the dorsolateral region of the AL. We traced the dorsolateral bundle of Wnt5 ${ }^{+}$fibers to a group of $\sim 20-30$ cells located $\sim 50 \mu \mathrm{m}$ anterior and lateral to the AL in the deutocerebrum (Fig. 2). Costaining with an antibody against the neuronal nuclear marker Elav showed that they were neurons (Fig. 2A). Costaining with antibodies against Acj6 and Drifter, which are expressed by subsets of PNs (Komiyama et al., 2003), showed that the cells express neither Acj6 nor Drifter (Fig. 2B,C). Furthermore, we induced wnt5 mutant PN clones using the mosaic analysis with a repressible cell marker (MARCM) technique (Lee and Luo, 1999). ALs containing large patches of mutant PNs developed normally (data not shown) suggesting that the PNs were unlikely to be the source of Wnt5, and that the PN-associated Wnt5 was generated by a different cell type. Because Wnt 5 acts cell nonautonomously, we could not conclusively eliminate PNs as a source of Wnt5. These results, together with the observation that these cells did not extend dendrites into the AL or axons to the mushroom body suggested that they were not PNs, but were a novel type of ALassociated neurons. We refer to them as dorsolateral cluster (DLC) neurons. In summary, we have identified a set of previously undescribed $\mathrm{Wnt} 5^{+}$cells that are tightly associated with the dorsolateral edge of the developing AL from $0 \mathrm{hAPF}$ to at least 36 hAPF, a period of major AL patterning.

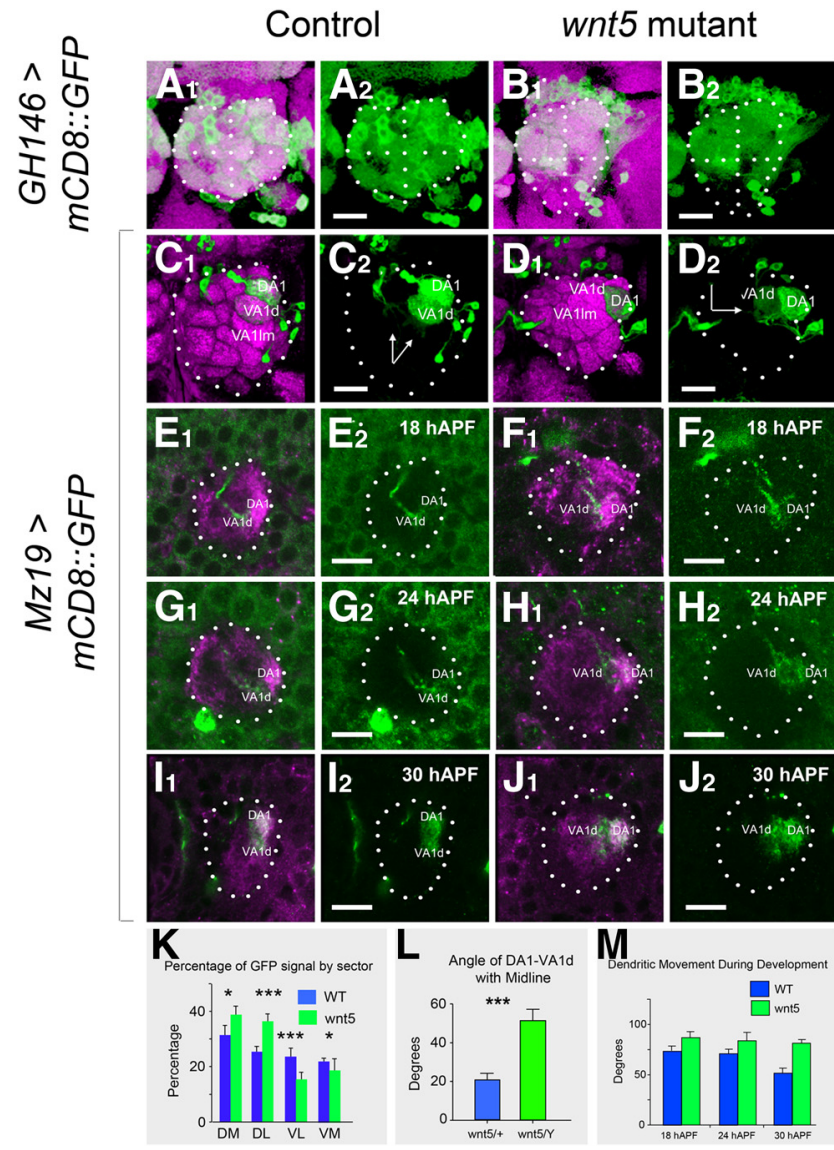

Figure 3. Ventral migration of PN dendrites is impaired in the wnt5 mutant. Adult control (A) and wnt5 mutant (B) ALs expressing UAS-mCD8::GFP under the control of GH146-Gal4 were stained with the nc $82 \mathrm{mAb}$ (magenta) and anti-CD8 (green) antibodies. Dotted lines highlight the boundaries of the ALs and bisect the ALs into quadrants. In the control, GH146 dendrites occupy all regions of the $A L$, but in the wnt5 mutant, the dendrites are absent from the ventral region of the AL. Adult control ( $($ ) and wnt5 mutant (D) ALs expressing UAS-mCD8::GFP under the control of Mz19-Gal4 were stained with nc82 (magenta) and anti-CD8 (green) antibodies. Arrows indicate the angle subtended by the line joining DA1 and VA1d glomeruli against the midline. The DA1, VA1d, and VA1Im dendrites are arranged dorsoventrally in the control AL, but mediolaterally in the wnt5 mutant AL. ALs of control $(\boldsymbol{E}, \boldsymbol{G}, \boldsymbol{I})$ and wnt5 mutant $(\boldsymbol{F}, \boldsymbol{H}, \boldsymbol{J})$ expressing UAS-mCD8::GFP under the control of Mz19-Gal4 were stained with anti-Drl (magenta) and anti-CD8 (green) antibodies at 18,24, and 30 hAPF. The VA1d dendrites migrated to a position ventral to DA1 in the control $(\boldsymbol{E}, \boldsymbol{G}, \boldsymbol{I})$, but failed to do so in the wnt5 mutant $(\boldsymbol{F}, \boldsymbol{H}, \boldsymbol{J})$. Quantification of the percentage of GFP staining in the different quadrants of the wild-type (blue) versus wnt5 mutant (green) ALs is shown in $K$. Quantification of the angle of VA1d-DA1 with the midline in the adult wnt5 mutant and control animals is shown in $\boldsymbol{L}$. Quantification of the angle of VA1d-DA1 with the midline at various times in pupal development of the wnt5 mutant and control animals is shown in $\boldsymbol{M}$. The angle decreased gradually in the wnt5 mutant but failed to do so in the control animal; ${ }^{* *} p<0.0001$. ${ }^{*} p<0.1$. Scale bars, $10 \mu \mathrm{m}$.

\section{Wnt5 is required for $\mathrm{PN}$ dendrites migration}

The expression of Wnt5 at a time when PN dendrites were actively pioneering the olfactory proto-map raised the possibility that Wnt5 might play a role in controlling PN dendrite development. To investigate this possibility, we examined the pattern of $\mathrm{PN}$ dendritic arbors in the wnt5 mutant using PN-specific markers. We first examined the dendritic pattern in the adult using the GH146-Gal4 marker. In control wnt5/+ heterozygotes, GH146 dendrites innervate $\sim 34$ of the $\sim 50 \mathrm{AL}$ glomeruli, creating a stereotyped pattern in the AL (Jefferis et al., 2001; Fig. 3A1,A2). Unlike in the control, where the ventral AL was substantially occupied by GH146 dendrites, the ventral AL in the wnt5 homozygote was poorly innervated by the dendrites (Fig. 3B1,B2). 
Quantifying the CD8 fluorescence intensities in each quadrant of the wnt5 mutant AL (DM, DL, VM, and VL) showed a decrease in the percentage of CD8 signal in the ventral AL $(25 \pm 7 \%$ in $w n t 5$, $n=6$, vs $42 \pm 5 \%$ in control, $n=6$, Student's $t$ test, $p<0.001)$ accompanied by an increase in the dorsal AL $(75 \pm 6 \%$ in $w n t 5$, $n=6$, vs $59 \pm 4 \%$ in control, $n=6, p<0.001$; Fig. $3 K)$. To further characterize this dorsal displacement of dendrites, we used the Mz19-Gal4 driver (Ito et al., 1998; Zhu and Luo, 2004) to label specific dendritic arbors (DA1, VA1d, and DC3). In the control, the VA1d arbors were located ventral to the DA1 arbors (Fig. 3C1,C2). In the wnt5 mutant, VA1d was located medial to DA1 instead, indicating that VA1d has displaced dorsally relative to DA1 (Fig. 3D1,D2). To accurately quantify the movements of the dendrites relative to each other, we measured the angle made by the sagittal midline with the line that joined DA1 and VA1d. In the control ALs the angle was $20.5^{\circ} \pm 3.8^{\circ}(n=9)$, whereas in the wnt5 mutant the angle was $51.7^{\circ} \pm 5.8^{\circ}(n=9, p=$ 0.0004; Fig. $3 L$ ). Our analyses showed that, in the absence of $w n t 5$ function, ventral dendrites were displaced to the dorsal region in the adult AL.

To investigate how the dendritic displacements occurred in the wnt5 mutant, we examined the PN dendrites between 16 and 40 hAPF. We first examined a small subset of dendrites using the Mz19-Gal4 driver. The DA1 and VA1d dendrites could be easily distinguished by their inputs (adPNs or lPNs), and their differential staining by the Drl antibody. We first characterized their changes in the wnt5/+ control. At $18 \mathrm{hAPF}$, the VAld dendrites were positioned medial and slightly ventral to the DA1 dendrites (Fig. 3E1,E2). Between 18 and 30 hAPF, the VA1d dendrites migrated ventrally, eventually ending up ventral to the DA1 dendrites (Fig. 3G1,G2,I1,I2). We quantified this movement by again measuring the angle made by the sagittal midline with the line that joined DA1 and VA1d. At $18 \mathrm{hAPF}$ the angle was $72.9^{\circ} \pm 4.9^{\circ}$ $(n=12)$ and at $30 \mathrm{hAPF}$ the angle was $51.7^{\circ} \pm 5.1^{\circ}(n=9)$. Thus, the VAld dendrites migrated $\sim 20^{\circ}$ around the DA1 dendrites between 18 and 30 hAPF in the wnt5/+ control (Fig. 3M). Examination of the wnt5 mutant ALs at these time points showed that this movement was impaired. From 18 to $30 \mathrm{hAPF}$, the VA1d dendrites migrated very little from their position medial to the DA1 dendrites (Fig. $3 F, H, I$ ). Quantification showed a change in angle of only $\sim 5^{\circ}$, from $86.7^{\circ} \pm 6.0^{\circ}(n=9)$ at $18 \mathrm{hAPF}$ to $81.5^{\circ}$ $\pm 3.3^{\circ}(n=10)$ at $30 \mathrm{hAPF}$ (Fig. $\left.3 M\right)$. Thus, in the absence of wnt5 function, ventral migration of the VA1d dendrites was strongly impaired. To determine whether the movement we observed in the Mz19 dendrites was exhibited by other dendrites, we labeled a large subset of the PN dendrites using the GH146-Gal4 driver. Tracking of a group of GH146 dendrites in the wnt5/+ control between 16 and $30 \mathrm{hAPF}$ revealed the same pattern of dendritic movement as that seen with the Mz19 dendrites (data not shown). Again, in the wnt5 mutant, the dendritic movements were strongly impaired relative to controls (angular movement of $\sim 15^{\circ}$ in wnt5 compared with $\sim 40^{\circ}$ in wnt5/+). In summary, ventral dendrites in the wnt5 mutant, such as VAld, failed to undergo normal ventromedial migration during development resulting in their apparent dorsal displacement in the mutant adult.

\section{Dorsolateral source of Wnt5 promotes ventral migration of PN dendrites}

From 16 to 30 hAPF Wnt5 is concentrated in two domains in the $\mathrm{AL}$, a dorsolateral and a ventrolateral domain, associated with wnt5-expressing DLC neurons and ORNs respectively. To assess the roles of the two cell types in the PN dendritic migration, we

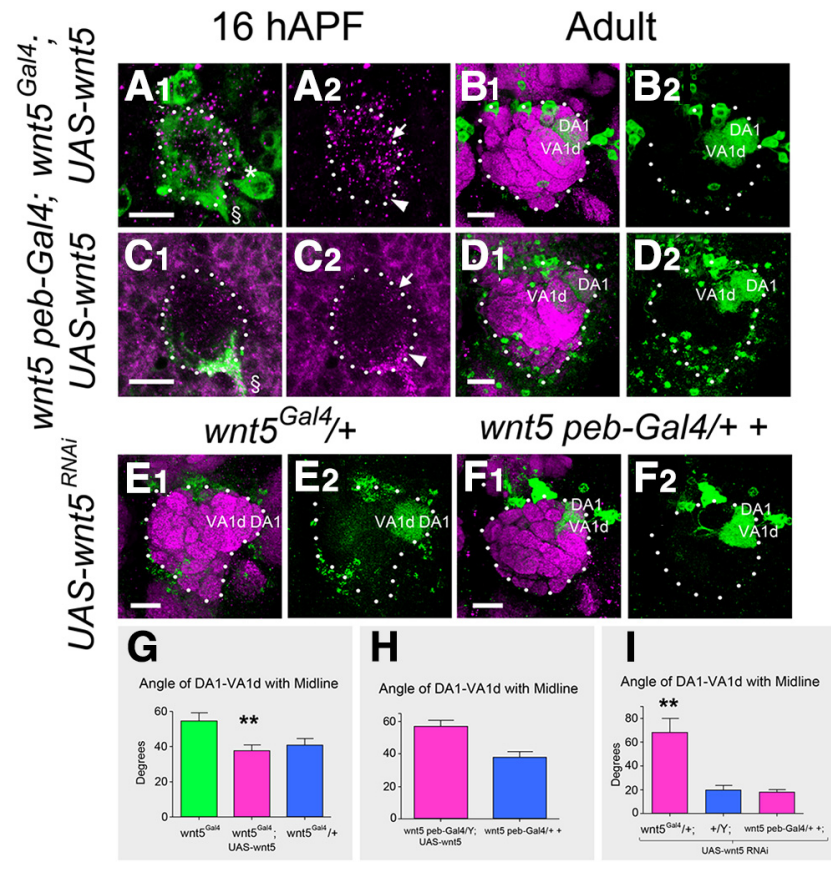

Figure 4. Wnt5 expressed from DLC neurons is necessary for PN dendritic migration. A pupal AL from hemizygous wnt $5^{\text {Gal4 }}$ mutant expressing UAS-wnt5 and UAS-mCD8::GFP was stained with anti-Wnt5 (magenta) and anti-CD8 (green) to visualize the position of the Wnt5 protein at $16 \mathrm{hAPF}(\boldsymbol{A 1}, \boldsymbol{A 2})$. Arrow indicates the dorsolateral AL, arrowhead the ventrolateral AL, asterisk the DLC nerve, and $\S$ the antennal nerve. The Wnt5 protein was restored primarily to the dorsolateral domain of the AL. An adult AL from hemizygous wnt $5^{\text {Gal4 }}$ mutant expressing UASwnt5 and Mz19-mCD8::GFP was stained with nc82 (magenta) and anti-CD8 (green) to assess AL development (B1). Restoration of Wnt5 to the dorsolateral domain of the AL strongly rescued AL development as determined by its wild-type shape and the orientation of the $M z 19$ dendrites (B2). A pupal AL from hemizygous peb-Gal4 wnt5 mutant expressing UAS-wnt5 and UAS-mCD8::GFP was stained with anti-Wnt5 (magenta) and anti-CD8 (green) to visualize the position of the Wnt5 protein at $16 \mathrm{hAPF}(\mathbf{C} 1, \mathbf{C 2})$. The Wnt5 protein was restored to the ventrolateral domain of the AL. An adult AL from hemizygous peb-Gal4 wnt5 mutant expressing UAS-wnt5 and Mz19-mCD8::GFP was stained with nc82 (magenta) and anti-CD8 (green) to assess AL development $(\boldsymbol{D})$. Restoration of Wnt 5 to the ventrolateral domain of the AL failed to rescue $A L$ development as determined by its elongated shape and the orientation of the $M z 19$ dendrites (D2). Adult ALs from animals expressing UAS-wnt $5^{R N A i}$ in the heterozygous wnt $5^{\text {Gal4 }} /+; M z 19-m C D 8:: G F P(\boldsymbol{E})$ or heterozygous wnt $5^{400}$ peb-Gal4/2+ $;$ Mz19-mCD8::GFP (F) backgrounds were stained with nc82 (magenta) and anti-CD8 (green) to assess AL development. Knock down of wnt5 in the DLC neurons resulted in a strong wnt5 loss-of-function AL phenotype, as judged from its elongated shape and orientation of the $M z 19$ dendrites $(\boldsymbol{E} 1, \boldsymbol{E} 2)$. Knock down of wnt5 in the ORNs had no effect on AL development, as judged from its wild-type shape and orientation of the $M z 19$ dendrites $(\boldsymbol{F 1}, \boldsymbol{F 2})$. Quantification of the angle of DA1-VA1d with the midline in the hemizygous wnt $5^{\text {Gal }}$ mutant expressing UAS-wnt5 showed strong rescue of AL development $(\boldsymbol{G})$. Quantification of the angle of DA1-VA1d with the midline in the hemizygous peb-Gal4 wnt5 mutant expressing UAS-wnt5 showed no rescue of AL development $(\boldsymbol{H})$. Quantification of the angle of DA1-VA1d with the midline showed that knock down of wnt5 in the DLC neurons (with wnt $5^{\text {Gal4 }}$ ) but not the ORNs (with peb-Gal4) strongly impaired AL development $(I) ;{ }^{* *} p<0.001$. Scale bars, $10 \mu \mathrm{m}$.

altered $w n t 5$ expression in the cells using the $w n t 5^{\text {Gal }}$ and pebGal4 drivers. We first expressed UAS-wnt5 under the control of either $w n t 5^{\text {Gal4 }}$ or peb-Gal4 in the wnt5 mutant and stained the ALs with the Wnt5 antibody to visualize the localization of the Wnt5 protein in the ALs. We observed that Wnt5 was found primarily in the anterior dorsolateral domain when expressed under the control of $w n t 5^{\text {Gal }}$ (Fig. $4 A 1, A 2$ ). When driven by peb-Gal4, which is specifically expressed in ORNs, Wnt5 is specifically localized to the posterior ventrolateral domain (Fig. $4 C 1, C 2)$. To determine whether the expression of Wnt5 in the dorsolateral or ventrolateral domain rescued AL development of the wnt5 mutant, we examined the adult AL structure by staining 
with the anti-Bruchpilot antibody (nc82) and labeling with the Mz19-mCD8::GFP transgene. Restoration of Wnt5 to the dorsolateral domain strongly rescued AL development, as assessed by the spherical shape of the AL and wild-type orientation of the $M z 19$ dendrites $\left(38.0^{\circ} \pm 3.4^{\circ}, n=10\right.$ vs $54.7^{\circ} \pm 4.6^{\circ}$ in $w n t 5^{\text {Gal }}$ mutant, $n=10, p=0.001$; Fig. $4 B 1, B 2, G)$. In contrast, restoration of Wnt5 to the ventrolateral domain failed to rescue AL development, as assessed by the elongated shape of the $\mathrm{AL}$ and abnormal orientation of the $M z 19$ dendrites $\left(57.9^{\circ} \pm 3.7^{\circ}, n=14\right.$ vs $51.7^{\circ} \pm 5.8^{\circ}$ in $w n t 5, n=12, p=0.36$; Fig. $\left.4 D 1, D 2, H\right)$. In a complementary approach, we used RNA interference to knockdown wnt5 expression in the heterozygous wnt5 background in either the DLC cells and ORNs, or the ORNs alone. A strong wnt5 mutant phenotype was induced when UAS-wnt $5^{R N A i}$ was expressed under the control of the $w n t 5^{\text {Gal4 }}$ driver but not the pebGal4 driver (Fig. 4E,F,I). Together, the genetic rescue and ablation results suggest that Wnt5, originating from the anterior dorsolateral domain but not the posterior ventrolateral domain, plays a role in regulating the targeting of $\mathrm{PN}$ dendrites and the development of the AL.

\section{Wnt5 overexpression disrupts PN dendritic targeting}

Our observation of a localized expression of Wnt5 in the dorsolateral AL suggests that the precise pattern of expression may be important in directing the targeting of the PN dendrites. To test this idea, we misexpressed Wnt5 in subsets of PNs and examined its effects on dendritic patterning. We first used the MARCM technique to drive the UAS-wnt5 transgene in the anterodorsal PNs (adPNs), lateral PNs (lPNs), and ventral PNs (vPNs) respectively. Each dendritic class formed a stereotyped and distinctive innervation pattern in the wild-type controls (Fig. 5A1-A4). Expression of Wnt5 in vPNs and DL1 PNs did not alter their dendritic pattern, probably due to low levels of GH146-Gal4 expression in those PNs (Fig. 5B3,B4). In contrast, expression of Wnt5 in the adPNs and lPNs significantly disrupted their dendritic innervation patterns. This was accompanied by severe distortion of the $\mathrm{AL}$ and derangement of the overall glomerular map, underscoring Wnt5's potent cell nonautonomous effects (Fig. 5B1,B2). Unfortunately, the severe distortion of the glomerular map prevented us from characterizing the targeting defects of the Wnt5 mis-expressing dendrites. To circumvent this problem, we used the Mz19-GAL4 driver to express Wnt5 only in the DA1, VA1d, and DC3 neurons. When expressed in these neurons, Wnt5 was transported to the dendrites, severely altering Wnt5's expression pattern in the $16 \mathrm{hAPF}$ AL (Fig. $5 E, F$ ). In the adult wild-type, DA1 is normally located adjacent and dorsal to VA1d, while DC3 is located posterior to VA1d and thus not visible on the AL surface (Fig. 5C). When Wnt5 was expressed in the Mz19 dendrites, DA1 frequently separated from VA1d and migrated ventrally, while DC3 frequently migrated to the AL surface (Fig. 5D). In some brains, the $M z 19$ dendrites were seen to inappropriately innervate the DA4 and D glomeruli (Fig. 5D). Our results therefore showed that wnt5 is both necessary and sufficient to direct PN dendritic movement and the precise pattern of Wnt5 expression in the AL is important in determining the proper targeting of the $\mathrm{PN}$ dendrites.

\section{Drl is expressed in a gradient by $\mathrm{PN}$ dendrites}

The receptor mediating Wnt5 signaling in the PN dendrites is unknown. The Drl atypical receptor tyrosine kinase has been shown to bind to Wnt5 and mediate its signaling in the migration of a number of cell types, including commissural axons, muscle fibers, and salivary gland cells (Callahan et al., 1996; Yoshikawa et
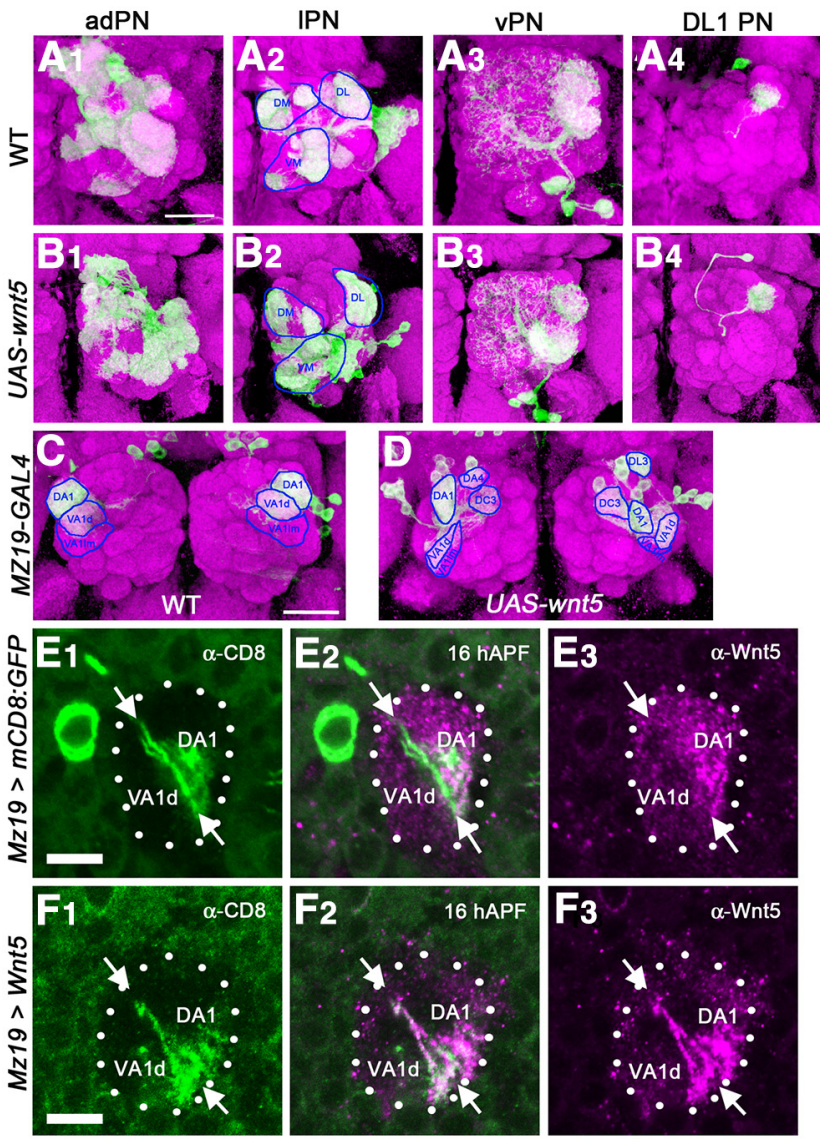

Figure 5. Mis-expression of Wnt5 in the PNs disrupts their dendritic targeting. The MARCM technique was used to label the adPNs, IPNs, vPNs, and DL1 PNs in the wild-type background (A1-A4), or simultaneously label and overexpress Wnt5 in the same PNs (B1-B4) in an otherwise wild-type background. Wild-type PN dendrites from each subclass displayed a stereotyped innervation pattern $(\boldsymbol{A} 1-\boldsymbol{A} 4)$. For example, adPNs target VA1d and VA1Im (A1), IPNs target DA1, VA7m, and VA5 (A2), vPNs target VA1Im and DA1 (A3) whereas DL1 PNs target the dorsolateral $\mathrm{AL}(\boldsymbol{A} 4)$. PN dendrites of the same subclasses overexpressing Wnt5 showed strong mis-targeting phenotypes (B1-B4, compare with $\boldsymbol{A} \mathbf{1}-\boldsymbol{A} 4)$. The adPNs (B1) and IPNs (B2) failed to display the stereotypical glomerular projection patterns seen in the wild-type. However, due to the severe deformation of the AL the exact nature of the derangements could not be determined. The vPNs (B3) and DL1 PNs (B4) showed normal targeting pattern. In a complementary approach, the $\mathrm{Mz} 19-\mathrm{Gal} 4$ driver was used to either label a subset of PN dendrites in the wild-type background $(\boldsymbol{C})$, or simultaneously label and overexpress Wnt5 (D) in the same PNs in an otherwise wild-type background. In the wild-type, DA1 is directly adjacent and dorsal to VA1d. DC3 is located more posteriorly and therefore not visible (C). Overexpression of Wnt5 in the DA1, VA1d and DC3 PNs led to strong mistargeting of these dendrites (Fig. 4, compare $\mathbf{D}, \mathbf{C}$ ). DA1 dendrites separated from VA1d dendrites and migrated ventrally next to VA1d. DC3 migrated anteriorly becoming visible on the surface of the AL. ALs expressing either UAS-mCD8::GFP (E), or UAS-mCD8::GFP and UAS-Wnt5 (F) under the control of Mz19-Gal4 were stained with anti-Wnt5 (magenta) and anti-CD8 (green) at 16 hAPF. In the control (E), DA1 dendrites reside within the dorsolateral domain of high Wnt5 concentration, whereas VA1d dendrites (arrows) lie at the edge of the domain, in a region of low Wnt5 concentration. When Wnt5 is mis-expressed under the control of Mz19-Gal4 $(\boldsymbol{F})$, the Wnt5 protein was transported to the dendrites, strongly altering the expression pattern of Wnt5 in the AL neuropil. Scale bars, $50 \mu \mathrm{m}$

al., 2003; Fradkin et al., 2004; Harris and Beckendorf, 2007; Lahaye et al., 2012). To determine whether Drl was expressed in the developing AL, we stained 16 hAPF ALs with antibodies against Drl and N-Cadherin proteins (Fig. 6A1,A2). Drl was highly expressed in the AL neuropil with an apparent high concentration in the dorsolateral AL (Fig. 6B1,B2). Quantification of the concentrations of Drl in a focal plane at $6 \mu \mathrm{m}$ from the anterior surface showed that Drl formed a DL $>$ VM gradient in the 

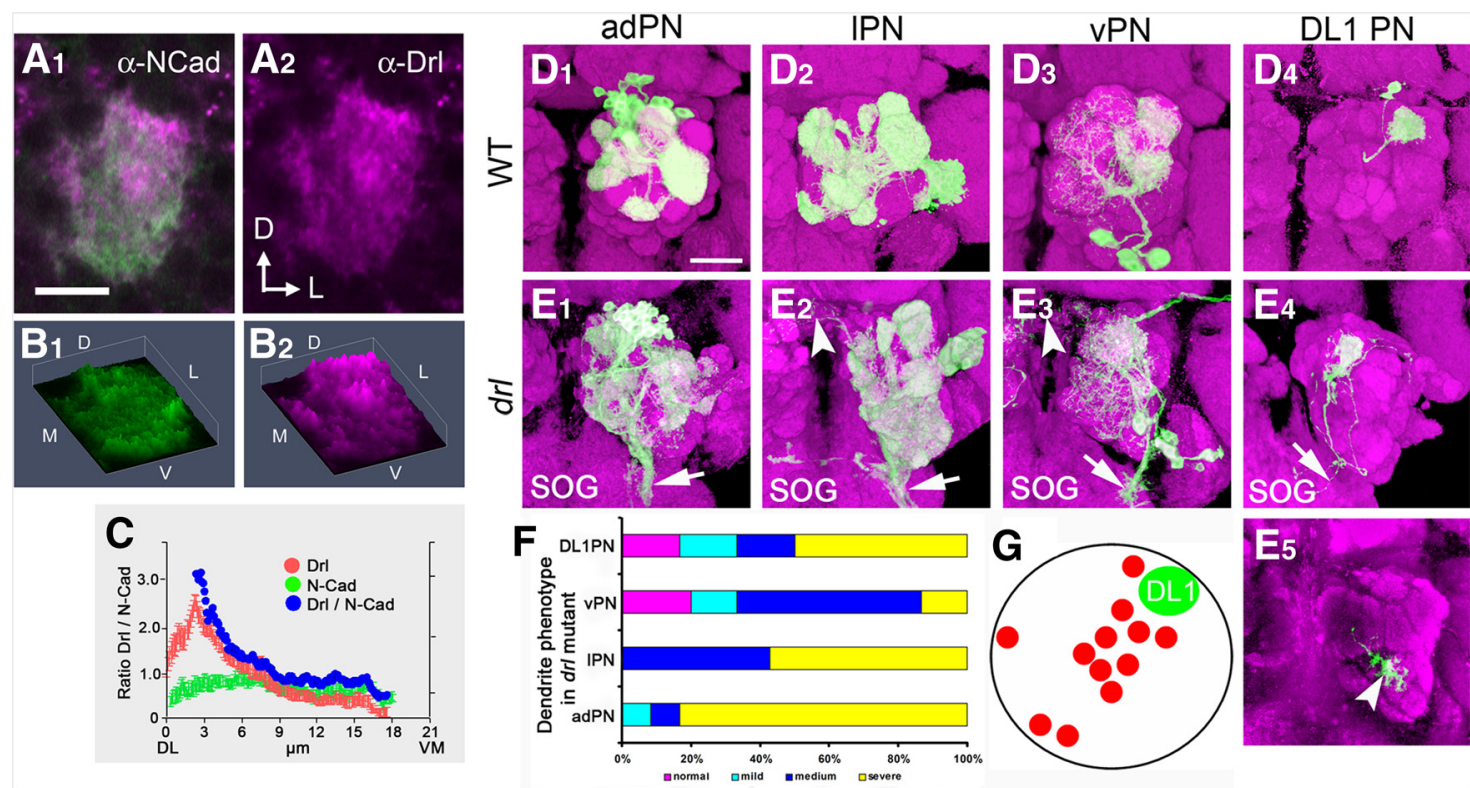

Figure 6. dr/mutation disrupts the stereotyped pattern of PN dendrites in the adult. A representative wild-type $16 \mathrm{hAPF}$ AL stained with antibodies against N-Cadherin (green) and Drl (magenta), showing that the Drl protein is concentrated at the dorsolateral region of the AL (A1, A2). 3-D histogram plots of the pixel intensities of the N-Cadherin (green) and Drl (magenta) proteins from $\boldsymbol{A}$, showing that Drl forms a decreasing DL $>V M$ gradient $(\boldsymbol{B 1}, \mathbf{B 2}$ ). A plot of the average $\mathrm{N}$-Cadherin (green) and Drl (red) pixel intensities, as well as normalized Drl values (blue) along the DL-VM axis of the AL, showing that DRL forms a gradient with the highest concentration at the dorsolateral pole (C). The MARCM technique was used to visualize subsets of PN dendrites in the adult wild-type and dr/ mutant brains. Dendrites of adPNs, IPNs, vPNs, and DL1 PNs exhibited stereotyped glomerular targeting pattern in the wild-type (D1-D4). For example, adPN dendrites target the VA1d and VA1Im glomeruli (A1), IPN dendrites target the DA1, DM2, and VA7m glomeuli (D2), vPN dendrites target the DA1 and VA1Im glomeruli (D3), whereas DL1 PN dendrites target the dorsolateral AL (D4). In drl homozygotes, these characteristic targeting patterns were not seen and glomerular boundaries were no longer easily distinguishable. The severity of the mis-targeting made characterization of the defects difficult (E1-E4). Remarkably, the PN dendrites projected abnormally to the contralateral ALs (arrowheads) and the subesophageal ganglion (E1-E3, arrows). Although DL1 dendrites arborized correctly in the dorsolateral AL, they frequently extend a branch to the ventromedial AL (E4, E5, arrows). Quantification of dendritic targeting defects of the PN subsets in the drl mutant $(\boldsymbol{F})$. Normal: magenta; mild: cyan, slight ectopic targeting or loss of targeting; medium: blue, half to one-third of dendrites show loss or ectopic targeting; severe: yellow, more than half of dendrites show aberrant phenotypes. Schematic summarizing the ectopic ventromedial targeting displayed by DL1 dendrites in the drl mutant (G). Red circles mark the ectopic termination sites of 12 independent single-cell DL1 clones. The Green circle marks the position of the DL1 arbor. Scale bars: $\boldsymbol{A}, 10 \mu \mathrm{m} ; \boldsymbol{D}, 50 \mu \mathrm{m}$.

16 hAPF AL (Fig. 6C). Close examination of the Drl staining suggested that Drl is present on $\mathrm{PN}$ dendrites. Indeed, staining of ALs expressing UAS-mCD8::GFP under the control of GH146Gal4 revealed extensive overlap between CD8 and Drl immunoreactivity, indicating that Drl was likely expressed by the PN dendrites (data not shown). Interestingly, the staining pattern of Drl showed remarkable resemblance to the staining pattern of Wnt5 (Fig. 6A1,A2, compared with Fig. 1B1,B2), suggesting that the two proteins might functionally interact on the PN dendrites. Drl was not expressed by all dendrites, as certain arbors, such as VA1d (Fig. 3E, G,I) were poorly stained, or unstained by the Drl antibody. MARCM labeling of the adPNs, IPNs, and vPNs demonstrated that Drl was expressed by the descendants of the anterodorsal, lateral, and ventral neuroblast lineages (data not shown). In summary, Drl was expressed in a DL $>$ VM gradient in the early developing AL and appeared to colocalize with the Wnt5 protein on PN dendrites. These results support the notion that Drl is differentially expressed by PN dendrites and acts to control PN dendritic targeting in response to the repulsive Wnt5 signal.

\section{Drl promotes the dorsolateral targeting of PN dendrites}

To determine whether Drl was necessary for the patterning of the $\mathrm{PN}$ dendrites, we first examined the PN dendritic pattern in adults homozygous for a $d r l$-null mutation. We used the MARCM technique with the GH146-Gal4 marker to separately label the adPN, IPN, and vPN dendrites. In the wild-type, each dendritic class was arranged in a stereotyped and distinctive pattern (Fig. 6D1-D4). In contrast, the dendritic patterns were se- verely disrupted in the $d r l$ mutant. This was particularly evident in the pattern of the adPN and IPN dendrites (Fig. 6E1,E2, quantified in $F$ ). The dendritic arbors showed a tendency to displace to the posterior ventromedial aspect of the AL, a region normally devoid of GH146-GAL4-labeled dendritic processes. In a subset of brains, adPNs, IPNs, and vPNs dendrites were even observed to project to the contralateral AL and subesophageal ganglion (Fig. $6 E 1-E 3$, arrows and arrowheads). Labeling of single DL1 neurons revealed that, although a main dendritic arbor was located in the dorsolateral $\mathrm{AL}$ as in the wild-type, a small branch was often found in the ventromedial aspect of the AL (Fig. 6E4,E5, summarized in $G$ ). We thus conclude that Drl is necessary for proper dendritic patterning.

We next examined the adPN, IPN, and DL1 dendrites of the drl-null mutant at different times during pupal development. In the wild-type, dendrites of each PN class occupied a restricted region of the AL at $16 \mathrm{hAPF}$ and gradually mature over 24 and 36 hAPF to produce the characteristic adult pattern at $50 \mathrm{hAPF}$ (Fig. $7 A, C, E)$. In the $d r l$ mutant, the adPN, IPN, and DL1 dendritic patterns were clearly aberrant at 24 and $36 \mathrm{hAPF}$, during the period of active dendritic migration (Fig. $7 B, D, F$ ). We observed the presence of ectopic dendrites in ventral and ventromedial regions of the AL. This phenotype was particularly evident in the DL1 dendrites, which normally innervate the dorsolateral AL (Fig. $7 E, F$ ). Although we observed disruptions in dendritic pattern as early as 16 hAPF (data not shown), most of the mutant phenotypes became apparent between 24 and 36 hAPF. These developmental studies therefore revealed that Drl functions dur- 


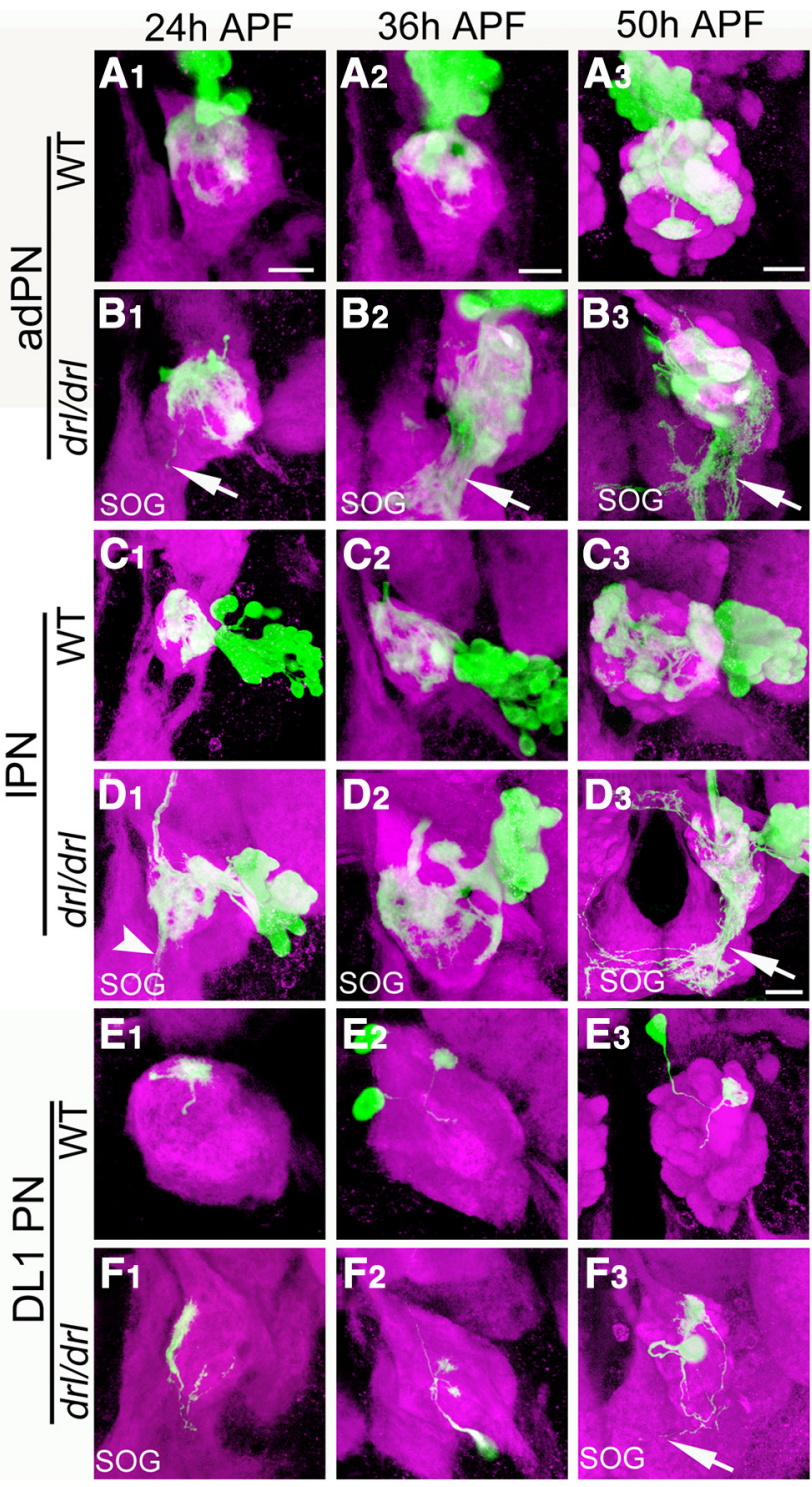

Figure 7. Drl is required for PN dendritic targeting between 24 and $50 \mathrm{hAPF}$ of development. The MARCM technique was used to visualize subsets of PN dendrites at the three indicated stages of pupal development in wild-type $(\boldsymbol{A}, \boldsymbol{C}, \boldsymbol{E})$ and $d r /$ mutant $(\boldsymbol{B}, \boldsymbol{D}, \boldsymbol{F})$ animals. Brains were stained with antibodies against $\mathrm{N}$-Cadherin (magenta) and CD8 (green); merged images are shown. In the wild-type, adPN dendrites target characteristic areas of the AL from 24 to $50 \mathrm{hAPF}$ (A1-A3). In the drl mutant, the adPN dendrites failed to establish this pattern. Importantly, dendrites could be seen projecting into the subesophageal ganglion (SOG) a phenotype not seen in the wild-type (B1-B3, arrow). Although the IPN dendrites project to characteristic regions in the wild-type $\left(C_{1-C}\right)$, they failed to do so in the $d r l$ mutant but project to the ventromedial AL (D1-D3, arrows). DL1 dendrites normally innervated the dorsolateral position of the AL (E1-E3). In the drl mutant, DL1 dendrites ectopically target the ventromedial region of the AL (F1-F3). Scale bars, $10 \mu \mathrm{m}$.

ing the period of active $\mathrm{PN}$ dendritic migration to promote the dorsolateral targeting of the PN dendrites.

\section{Drl functions autonomously in PNs}

The localization of Drl immunoreactivity to PN dendrites suggested that $d r l$ functioned in the dendrites to regulate their targeting during AL development. To test this hypothesis, we generated homozygous $d r l$ mutant clones of adPNs, lPNs, vPNs, and DL1 PNs in the $d r l$ heterozygous background and assessed the effects on dendritic migration. We observed that homozygosity for $d r l$ frequently resulted in the displacement of dendrites to
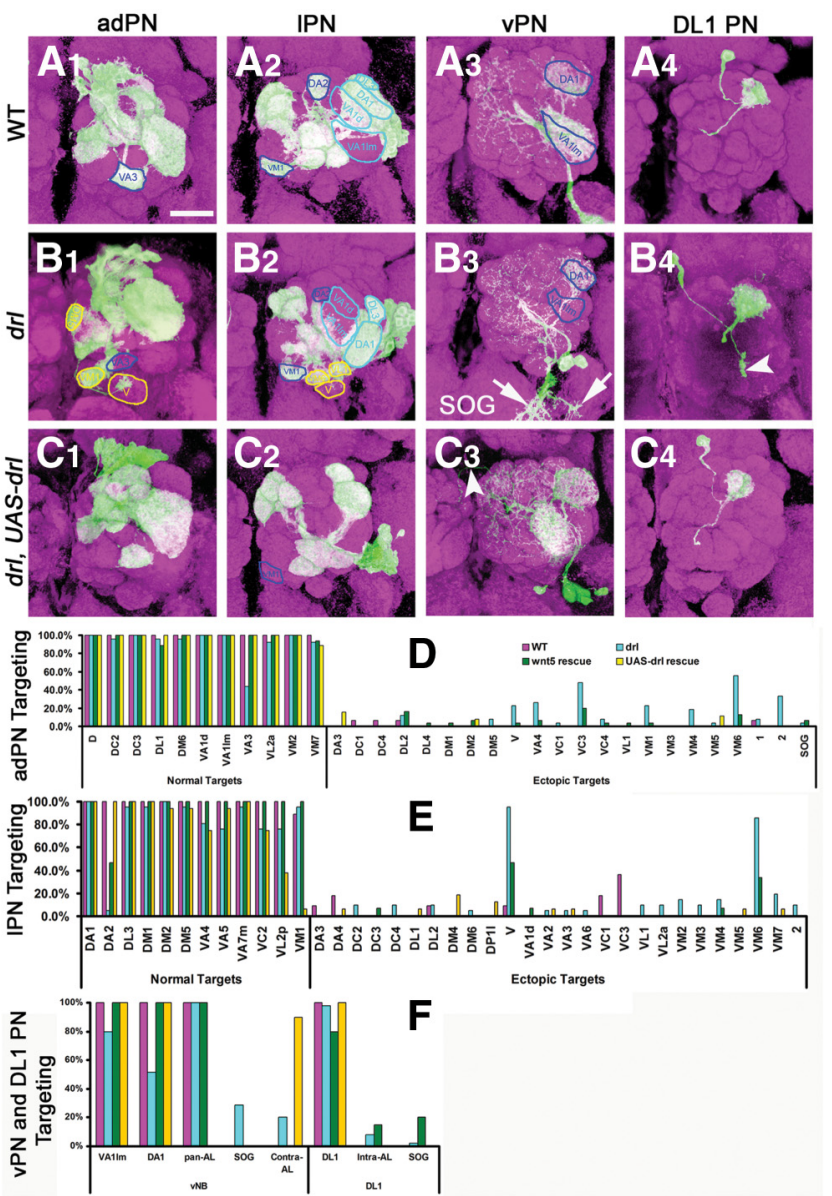

Figure 8. $d r l$ functions autonomously in PNs to antagonize wnt5 signaling. The MARCM technique was used to eliminate $d r l$ function in clones of PNs in an otherwise drl heterozygous background to assess the requirement of $d r l$ in the PNs. Adult ALs were stained with nc 82 (magenta) and anti-CD8 (green) to visualize the neuropil and PN clones; merged images are shown. Clones of wild-type adPNs, IPNs, vPNs, and DL1 PNs showed normal glomerular targeting (A1-A4). Clones of homozygous drl mutant adPNs, IPNs, vPNs, and DL1 PNs showed severely disrupted dendritic targeting (B1-B4). drl mutant adPNs failed to target VA3 (compare $B 1$ with $A 1$, blue outlines) and instead mis-target to DM5, VM1 and V ( $B 1$, yellow outlines). $d r l$ mutant IPNs failed to target their normal DA2 regions (compare $\boldsymbol{B} 2$ with $\boldsymbol{A 2}$, dark blue outlines) and instead mistarget to VM6, VL1, and V ( $B 2$, yellow outlines). The position of DA1 was also shifted ventrally (light blue outlines). drI mutant vPNs failed to target VA1Im and DA1 (compare $B 3$ to $\mathbf{A 3}$ ) and even project their dendrites to the subesophageal ganglion (B3, arrows). $d r l$ mutant DL1 dendrites mistarget to ventral part of the AL (B4, arrowhead). Restoration of $d r l$ specifically in the PNs rescued their ability to target their cognate glomeruli, but also produced some gain-of-function phenotypes ( $\mathbf{C}$ - $\mathbf{C} 4$ ). For example, IPNs failed to innervate VM1 (compare $\mathbf{C}$ with $\boldsymbol{A} \mathbf{2}$ and $\boldsymbol{B} \mathbf{2}$, blue outlines) and vPNs extend dendrites to the contralateral AL (C3, arrowhead). Quantification of PN dendritic defects in the various genotypes is shown in $\mathbf{D}-\boldsymbol{F}$. Magenta: wild-type clones; cyan: $d r l$ mutant clones; green: $d r l$ mutant clones in the heterozygous wnt 5 background; yellow: drl mutant clones expressing UAS-drl. Scale bars, $50 \mu \mathrm{m}$.

ventromedial sites (Fig. 8A1-A4, compare with B1-B4). For example, the DA1 dendrites, which are normally located dorsal to VA1d and VA1lm, migrated ventrally to a position next to VA1lm (Fig. 8A2, compare with B2). Another dorsal dendrite, DA2, was absent in $\sim 95 \%$ of ALs, whereas a ventral dendrite, VA3, was absent in $\sim 50 \%$ of ALs (Fig. $8 A 1$, compare with B1, quantified in $D-F)$. Multiglomerular vPNs also failed to innervate their dorsal targets, VA1lm and DA1 (Fig. 8A3, compare with $B 3$, quantified in $F$ ). Clones of single DL1 PNs revealed that even as the mutant cells projected dendrites to ectopic locations, they frequently innervated their normal targets (Fig. 8A4, compare with B4). It is therefore likely that defects were more pervasive than those ob- 


\section{MARCM Labeling of pan-AL vPNs}
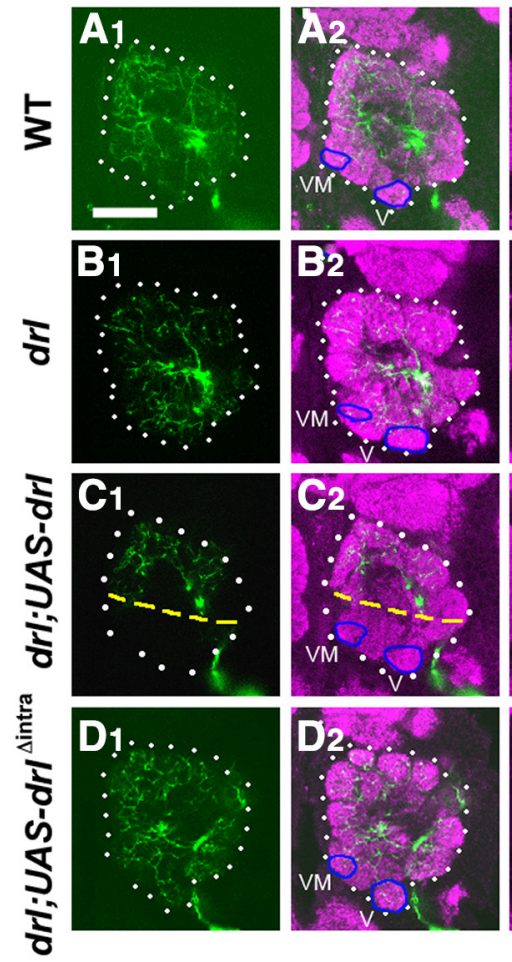
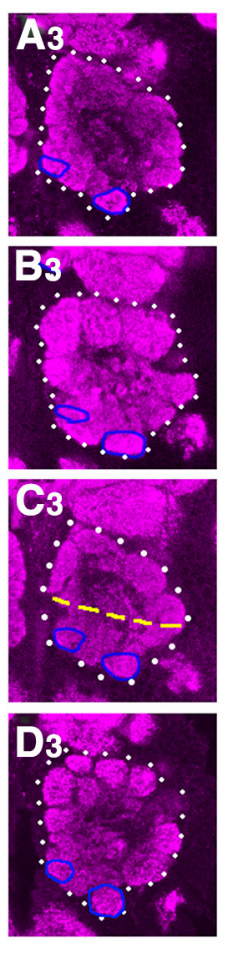

\section{GH146 Labeling of PN Dendrites}
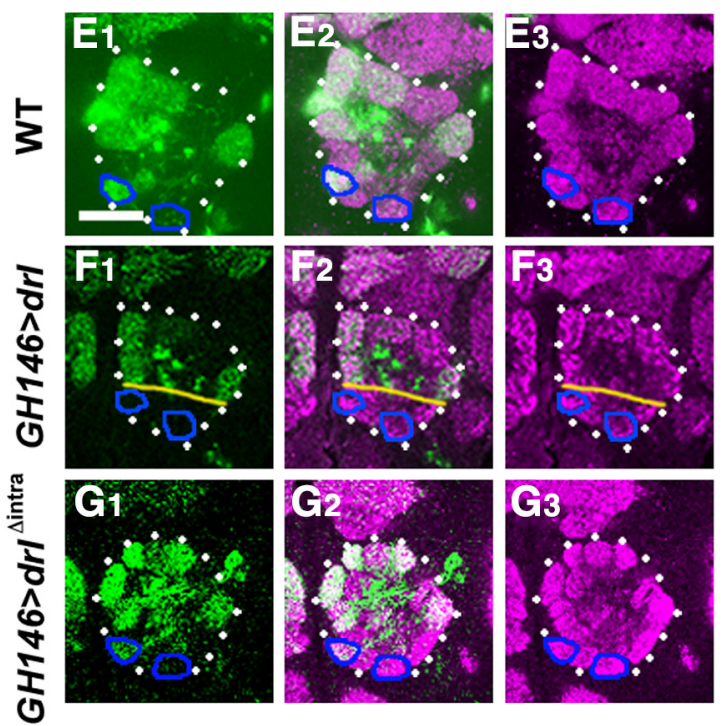

Figure 9. Overexpression of $d r l$ in PNs results in the loss of dendrites from the ventromedial AL. The MARCM technique was used to overexpress the UAS- $m$ CD8::GFP transgene alone, or simultaneously with UAS-drl transgenes in vPNs under the control of GH146-Gal4 (A-D). Comparable posterior sections are shown of adult ALs stained with anti-CD8 (green) to visualize the vPN clones and nc82 (magenta). White dotted lines highlight the ALs, blue circles highlight the V and VM1 glomeruli, and yellow dashed lines highlight the ventral limit of GH146-labeled dendrites. Pan-AL vPN dendrites arborize throughout the entire AL in the wild-type and $d r /$ mutant $(\boldsymbol{A}, \boldsymbol{B})$. vPNs overexpressing dr/ displayed reduced innervation of the ventromedial region of the AL ( $\boldsymbol{C}$ - $\mathbf{C}$ ). vPNs overexpressing drl $\Delta$ intra, encoding an intracellular domain truncated protein, showed normal innervation of the AL (D1-D3). The GH146-Gal4 driver was used to overexpress UAS-mCD8::GFP and either UAS-drl or UAS-drl $\Delta$ intra in the wild-type background $(\boldsymbol{E}-\boldsymbol{G}$ ). Comparable posterior sections are shown of adult ALs stained with nc82 mAb (magenta) and anti-CD8 (green). In the wild-type, GH146-labeled dendrites target ventromedial glomeruli including V and VM1 (E1-E3, blue outlines). Overexpression of $d r /$ in the PNs resulted in the loss of PN dendrites from the V and VM1 glomeruli (F1-F3). Overexpression of $d r l \Delta$ intra had no effect on the pattern of dendritic targeting compared with the wild-type (G1-G3). Scale bars, 50 $\mu \mathrm{m}$.

served by a loss of targeting alone. Indeed, 100\% of the mutant adPNs and IPNs displayed dendrites at ectopic sites. Although we were not able to assign class identity to the ectopic dendrites, quantification of the positions of the ectopic dendrites demonstrated their strong preference for targeting the posterior ventromedial AL (Fig. 8D-F). Restoration of $d r l$ function to the MARCM clones using the UAS- $d r l$ transgene substantially rescued the dendritic targeting, confirming that Drl acted in the PNs (Fig. 8C1-C4, quantified in $D-F$ ). Expression of the UAS-drl $l^{\Delta i n t r a}$ transgene, encoding a truncated form of Drl lacking the cytoplasmic domain, failed to rescue dendritic targeting, indicating that the cytoplasmic domain, and hence likely signal transduction by Drl, is critical for Drl function (data not shown). In summary, our results showed that $d r l$ functioned cell-autonomously in PNs to promote the targeting of their dendrites to the dorsolateral region of the AL. The differential requirement for $d r l$ among PNs suggests that $d r l$ does not function in all PNs and that other genes in addition to $w n t 5$ and $d r l$ may also regulate DL $>$ VM PN dendritic targeting.

\section{Overexpression of drl disrupts $\mathrm{PN}$ dendritic migration}

Interestingly, we observed the loss of some ventral dendrites, such as VL2p, VC2 and VA4 in the MARCM rescued animals in which $d r l$ function was expressed in subclasses of GH146 neurons (Fig. 8). This ventral loss of dendrites was particularly evident in the pan-AL arborizing vPNs, whose dendritic branches were ex- cluded from the ventromedial region of the AL (Fig. 9C, compare with $A$ ). We hypothesized that these defects were caused by inappropriate levels of Drl expression in dendrites that normally express little Drl. In support of this hypothesis, expression of $U A S$ - $d r l$ with the GH146-GAL4 driver in the wild-type background also disrupted the dendritic targeting in the ventromedial domain (Fig. 9E,F). The VM1 dendrites were absent, while the VA4, VC2 and VM3 dendrites were displaced more anteriorly at high frequencies (Fig. 9F, and data not shown). This gain-offunction effect required the Drl cytoplasmic domain, as misexpressing the UAS-drl ${ }^{\Delta i n t r a}$ transgene led to normal dendritic targeting (Fig. 9G, compare with E,F). Together, our results show that $d r l$ plays an instructive role in $\mathrm{PN}$ dendrites, where it acts to direct their migration toward the anterior and dorsal regions of the AL. The ability of Drl to control PN dendritic migration requires its cytoplasmic domain, suggesting that Drl transduces a signal upon binding its ligand.

\section{drl suppresses wnt5's function to promote dorsolateral movement of $\mathrm{PN}$ dendrites}

The colocalization of the Wnt5 and Drl proteins in the developing $\mathrm{AL}$ and the requirement for both in the development of the AL raised the question of how they might work together to regulate dendritic targeting. That the loss of $w n t 5$ led to the dorsolateral displacement of dendrites, while the loss of $d r l$ led to the opposite, ventromedial displacement of dendrites, suggested that 

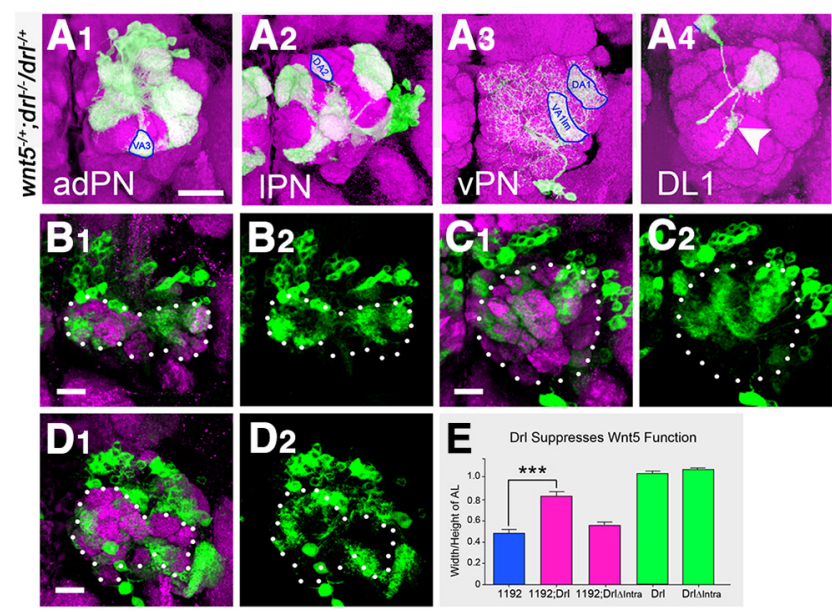

$\mathbf{F}$
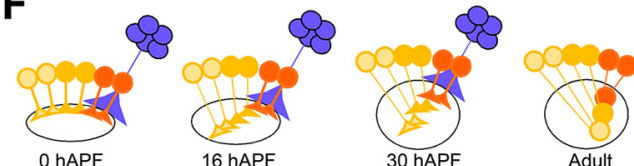

Figure 10. Drl antagonizes the repulsive function of Wnt5 in PN Dendrites. To assess genetic interaction between $d r l$ and wnt5, the MARCM technique was used to eliminate $d r l$ function in clones of PNs in the wnt5 heterozygous background. Adult ALs were stained with nc82 (magenta) and anti-CD8 (green) to visualize the neuropil and PN clones; merged images are shown. In the presence of half the dose of the wnt5 gene, $d r l$ mutant adPN, IPN, and vPN dendrites exhibited wild-type patterns of targeting (A1-A3, compare with Fig. $8 \boldsymbol{B} \mathbf{1}-\boldsymbol{B} \mathbf{4})$. Blue circles indicate wild-type targeting of select dendrites. DL1 dendrites still showed slight defects (A4, arrowhead). Thus, a reduction in wnt5 function strongly suppressed the $d r l$ loss-of-function mutant phenotype. Adult ALs from animals expressing UAS-wnt $5^{1192}$ under the control of GH146-Gal4 (B-D) were stained with nc82 (magenta) and anti-CD8 (green). Merged channels are shown on the left and green channels are shown on the right. Dotted lines highlight the ALs. Expression of UAS-wnt $5^{1192}$ by the PNs resulted in severe distortion of the ALs, with the ALS acquiring an abnormal dumbbell shape $(\boldsymbol{B} 1, \boldsymbol{B} 2)$. Coexpression of the UAS-drl transgene, encoding a full-length Drl protein, with UAS-wnt $5^{1192}$ strongly suppressed the wnt5 gain-offunction phenotype, restoring the $A L$ to a more wild-type shape $(\boldsymbol{C}, \mathbf{C} 2)$. Coexpression of the UAS-drIDintra transgene, encoding a truncated Drl protein, with UAS-wnt5 $5^{1192}$ did not suppress the wnt5 gain-of-function phenotype $(\boldsymbol{D} 1, \mathbf{D} 2)$. The effect of the overexpression of the wnt5 and $d r$ genes on AL morphology and their genetic interactions is quantified in $\boldsymbol{E}$. These genetic interaction data indicated that $d r l$ and wnt5 act antagonistically in PN dendritic migration. A model of how Wnt5 and Drl control PN dendritic migration is presented in $\boldsymbol{F}$. PNs express different levels of Drl (red, orange, yellow), which allow their dendrites to differentially respond to the DL $>$ VM Wnt5 gradient. The Wnt5 protein is deposited at the dorsolateral edge of the AL by DLC neurons (blue). PNs expressing high levels of Drl (red) are less repelled by Wnt5 and remain in the dorsolateral $A L$, whereas PNs expressing low levels of Drl (yellow) are strongly repelled by Wnt5 and migrate to the ventrolateral $\mathrm{AL} ;{ }^{* *} p<0.0001$. Scale bar, $10 \mu \mathrm{m}$.

the two genes act antagonistically. To test this hypothesis, we examined the interaction between the two genes using two complementary approaches. In the first approach, we asked whether elimination of a single copy of the wnt5 gene would modify the $d r l$ dendritic phenotype. We therefore induced clones of $d r l$ mutant $\mathrm{PN}$ in the heterozygous wnt5 mutant background and scored the phenotypes in the adult. Heterozygosity for wnt5 largely suppressed the dendritic targeting defects of the $d r l$ mutant adPNs, lPNs, and vPNs (Fig. 10A1-A4, compare with Fig. 8A1-B4, quantified in Fig. $8 D-F)$. Both the loss of normal dendritic targeting and ectopic ventromedial targeting were suppressed. Quantification of the adPN and IPN defects showed a threefold reduction in the $w n t 5 /+; d r l / d r l$ mutant compared with the $d r l / d r l$ mutant (Fig. $8 D, E$ ). Interestingly, heterozygosity of wnt5 slightly increased the penetrance of the $d r l$ DLl mistargeting phenotype (Fig. 8D). In the second approach, we asked whether coexpression of $d r l$ and wnt5 would suppress the wnt5 gain-of-function dendritic phenotype. We observed that expression of the UAS$w n t 5^{1192}$ transgene under the control of the GH146-Gal4 driver in the wild-type background led to severe distortion of the AL (Fig. 10B1-B2). The AL acquired a dumbbell shape (widened mediolaterally and narrowed dorsoventrally, ratio of height/width $=$ $0.48 \pm 0.04, n=28$; Fig. 10E). By contrast, expression of either the UAS-drl or UAS-drl $l^{\Delta i n t r a}$ transgene under the control of GH146-Gal4 did not disrupt the AL shape (Fig. 9F, G), with the ALs retaining normal aspect ratios of the wild-type (ratios = $1.09 \pm 0.01, n=13$ and $1.17 \pm 0.04, n=14$ respectively; Fig. $10 E)$. Coexpression of the UAS-wnt $5^{1192}$ transgene with the UAS$d r l$ transgene suppressed the AL distortion, returning the AL to a relatively wild-type shape (ratio $=0.83 \pm 0.04, n=26, p<$ 0.0001 ; Fig. 10C1,C2,E). Coexpression of the UAS-wnt5 $5^{1192}$ transgene with the UAS-drl ${ }^{\Delta \text { intra }}$ transgene however, had no effect on the wnt5 gain-of-function AL phenotype (Fig. 10D1,D2,E). Thus, our genetic interaction data indicated that $d r l$ antagonizes $w n t 5$ function to allow proper dorsolateral targeting of PN dendrites and AL development. The ability of Drl to antagonize Wnt5 signaling requires the Drl cytoplasmic domain suggesting that Drl suppresses Wnt5 via a downstream signaling mechanism.

\section{Discussion}

An important unanswered question in neuroscience is what are the developmental mechanisms that pattern neural maps. There are at least two hypotheses to explain the patterning of the olfactory map. The first is that neuronal processes of the map contain intrinsic information that directs their autonomous sorting through axon-axon or dendrodendritic interactions to create a prototypic map (Komiyama and Luo, 2006). The second is that extrinsic information from outside of the olfactory map directs its patterning (Komiyama et al., 2007; Sweeney et al., 2011). We addressed these possibilities by examining the patterning of the Drosophila PN dendrites, which play crucial roles in the initial establishment of the olfactory map. We show that the Wnt5 acts as an extrinsic cue that provide patterning information along the DL-VM axis of the fly olfactory map (Fig. 10F, model). We also show that Drl, a known Wnt5 receptor, is differentially expressed in $\mathrm{PN}$ dendrites and thus provides intrinsic information for dendritic sorting. By blocking the dendrite-repulsing activity of Wnt5, Drl allows PN dendrites to localize appropriately along the DL-VM axis.

We present the following pieces of evidence in support of such a model of AL patterning. First, the Wnt5 protein is expressed in a distinct pattern between 0 and $30 \mathrm{hAPF}$, a period of major PN dendritic growth and migration, being highly concentrated in the dorsolateral AL and declining to lower levels in central and ventromedial areas. We show that a likely source of Wnt5 is a cluster of exogenous neurons, the DLC neurons, located some $50 \mu \mathrm{m}$ lateral and anterior to the AL. These neurons extend long fibers, which terminate on the anterior, dorsolateral edge of the AL, where they deposit Wnt5 protein. We also find that Wnt5 is concentrated in the posterior, ventrolateral edge of the AL, associated with the ingrowing adult ORN axons. However, we showed that neither the selective expression of Wnt5 in the ventrolateral AL, nor the knock down of wnt5 in the ORNs, affected AL development, suggesting that Wnt5 provided by the ORNs does not play a major role in PN dendritic targeting. Our data therefore indicate that the developing $\mathrm{AL}$ receives patterning information from the extrinsic DLC neurons, which allows the olfactory map to be properly aligned with the major axes of the brain. Indeed, the loss of Wnt5 disrupts the alignment of the glomerular map relative to overall brain structure. 
Our developmental studies showed that Wnt 5 repels a subset of dendrites during the period between 16 and $30 \mathrm{hAPF}$. In the wild-type, PN dendrites have not achieved their final pattern at $16 \mathrm{hAPF}$, even though they are largely targeted to restricted regions of the AL. Ventromedial dendrites are located only medial to dorsolateral dendrites (Fig. 10F). In the ensuing $14 \mathrm{~h}$, the ventromedial dendrites migrate ventrally to their final fully ventral positions. Loss of wnt5 prevents the ventral migration of the ventromedial PN dendrites, indicating that Wnt5 acts as a repulsive cue for the dendrites. Thus, our results showed that Wnt5 does not participate in the initial segregation of PN dendrites to restricted domains (Jefferis et al., 2004), but instead in the rearrangement of dendritic arbors to their final positions between 16 and $30 \mathrm{hAPF}$. We propose that the Wnt 5 gradient provides positional information along the DL-VM axis, allowing PN dendrites expressing different levels of Drl to localize to their appropriate positions along this axis.

We and others have previously shown that the Drl protein is also necessary for the development of the fly olfactory map during the phase that it is pioneered by the ORN axons (Yao et al., 2007; Sakurai et al., 2009). Here we show that Drl also acts to pattern the PN dendritic map between 16 and 30 hAPF by modulating dendritic responses to the Wnt5 signal. At this stage, Drl is differentially expressed by the PN dendrites in a DL $>$ VM gradient across the AL. Loss of Drl caused dorsal dendrites to displace ventromedially, indicating that $\mathrm{Drl}$ acts to promote dorsal migration of dendrites. The opposite effects of Drl and Wnt5 on dendritic movement suggest that Drl and Wnt5 act antagonistically. Indeed, removal of a copy of the wnt5 gene strongly suppressed the mis-targeting defects displayed by the $d r l$ mutant dendrites, indicating that Drl promotes dorsolateral dendritic migration by blocking the dendrite-repulsing activity of Wnt5. How do different PN dendrites classes migrate to different positions in the Wnt5 gradient? The loss of Drl caused dorsal dendrites to displace ventromedially, whereas the overexpression of Drl caused ventral dendrites to displace dorsolaterally. We therefore propose that the level of Drl expression is instructive for targeting of the PN dendrites along the DL-VM axis. Dendrites expressing high levels of Drl are less repelled by Wnt5 and thus migrate dosolaterally, whereas dendrites expressing low levels of Drl are more repelled by Wnt 5 and thus migrate ventromedially. The pattern of Drl expression therefore provides AL-intrinsic information that directs dendritic targeting.

It is possible that Drl inhibits Wnt5 activity through simple sequestration of the Wnt5 protein via its WIF domain as we have shown for glial cell-derived Drl acting during the later stages of olfactory map patterning (Yao et al., 2007). However, our ectopic expression and rescue experiments revealed that Drl requires its intracellular kinase domain for function during PN dendrite patterning, suggesting that Drl actively transduces a signal to block Wnt5 activity. Thus, Drl likely displays different modes of function, sequestration versus signal transduction, in different cell types during the different phases of olfactory map patterning. Our observations that Drl antagonizes Wnt5 signaling raise the question as to the identity of the other PN Wnt5 receptor(s) that Drl modulates. A number of possibilities exist; particularly interesting is Drl-2, which has been shown to likely transduce a Wnt5 signal during formation of the ALs (Sakurai et al., 2009). Other possibilities include members of the Frizzled (Schulte and Bryja, 2007) and Ror (Green et al., 2008; Petrova et al., 2014) Wnt receptor families.

In our model, Wnt5 likely counteracts an opposing VM-DL force, as the loss of Wnt5 leads to the dorsolateral displacement of
PN dendrites. The identity of this opposing force is unknown. It is possible that it is mediated by the Sema- $2 \mathrm{a}$ and Sema- $2 \mathrm{~b}$ proteins. Sema-2a and Sema-2b are secreted by the degenerating larval AL, which is located directly opposite of the DLC cells, where they repel PN dendrites (Sweeney et al., 2011). Interestingly, the loss of Sema-2a and Sema-2b leads to the ventromedial displacement of PN dendrites, but the nature of the inferred DL-VM force was not explored. We propose that it is mediated by Wnt5. Thus, between 0 and $16 \mathrm{hAPF}$, the AL may contain two opposing gradients: a DL $>$ VM Wnt5 gradient and a DL $<$ VM Sema- $2 \mathrm{a} / 2 \mathrm{~b}$ gradient and these counter-gradients direct the patterning of the PN dendrites. Each dendrite would thus be guided by two repulsive cues: Wnt 5 from the DL pole and Sema-2a/2b from the VM pole. Final dendritic positions would be determined by the intersection of these gradients and would therefore depend on the relative expression levels of the ligands and their receptors at each point in the AL. Additional experiments will be needed to test this hypothesis.

We report in this paper a unique dendritic targeting phenomenon. The PN dendritic movements that we have described exhibit a circular directionality, with arbors moving around each other (Fig. 3; Sakurai et al., 2009). These movements initially seem difficult to reconcile with the orthogonal movements observed in other neural maps. We suggest that the circling movements are necessary in the spherical, relatively small AL, where dendrites must circumnavigate each other as they rearrange to create the final pattern. Rotation of cells, or groups of cells, to align with the axes of the body has previously been described in other tissues. Examples are hair cells on the fly wing, ommatidia in the fly eye, and hair follicles in the mouse epidermis (Tomlinson and Struhl, 1999; Devenport and Fuchs, 2008; Aigouy et al., 2010). In all the reported cases, the rotation events occur in epithelial tissues. To the best of our knowledge, our report describes for the first time the circular reordering of dendrites in the CNS. Elucidation of the Wnt5/Drl signaling pathway will shed light on the molecular and cellular underpinnings of this novel morphogenetic event.

\section{References}

Aigouy B, Farhadifar R, Staple DB, Sagner A, Röper JC, Jülicher F, Eaton S (2010) Cell flow reorients the axis of planar polarity in the wing epithelium of Drosophila. Cell 142:773-786. CrossRef Medline

Ang LH, Kim J, Stepensky V, Hing H (2003) Dock and Pak regulate olfactory axon pathfinding in Drosophila. Development 130:1307-1316. CrossRef Medline

Ang LH, Chen W, Yao Y, Ozawa R, Tao E, Yonekura J, Uemura T, Keshishian H, Hing H (2006) Lim kinase regulates the development of olfactory and neuromuscular synapses. Dev Biol 293:178-190. CrossRef Medline

Cadigan KM, Nusse R (1997) Wnt signaling: a common theme in animal development. Genes Dev 11:3286-3305. CrossRef Medline

Callahan CA, Bonkovsky JL, Scully AL, Thomas JB (1996) derailed is required for muscle attachment site selection in Drosophila. Development 122:2761-2767. Medline

Corty MM, Matthews BJ, Grueber WB (2009) Molecules and mechanisms of dendrite development in Drosophila. Development 136:1049-1061. CrossRef Medline

Couto A, Alenius M, Dickson BJ (2005) Molecular, anatomical, and functional organization of the Drosophila olfactory system. Curr Biol 15:15351547. CrossRef Medline

Devenport D, Fuchs E (2008) Planar polarization in embryonic epidermis orchestrates global asymmetric morphogenesis of hair follicles. Nat Cell Biol 10:1257-1268. CrossRef Medline

Fishilevich E, Vosshall LB (2005) Genetic and functional subdivision of the Drosophila antennal lobe. Curr Biol 15:1548-1553. CrossRef Medline

Fradkin LG, van Schie M, Wouda RR, de Jong A, Kamphorst JT, RadjkoemarBansraj M, Noordermeer JN (2004) The Drosophila Wnt5 protein me- 
diates selective axon fasciculation in the embryonic central nervous system. Dev Biol 272:362-375. CrossRef Medline

Fradkin LG, Dura JM, Noordermeer JN (2010) Ryks: new partners for Wnts in the developing and regenerating nervous system. Trends Neurosci 33: 84-92. CrossRef Medline

Gong WJ, Golic KG (2003) Ends-out, or replacement, gene targeting in Drosophila. Proc Nat Acad Sci U S A 100:2556-2561. CrossRef Medline

Green JL, Kuntz SG, Sternberg PW (2008) Ror receptor tyrosine kinases: orphans no more. Trends Cell Biol 18:536-544. CrossRef Medline

Harris KE, Beckendorf SK (2007) Different Wnt signals act through the frizzled and RYK receptors during Drosophila salivary gland migration. Development 134:2017-2025. CrossRef Medline

Ito K, Suzuki K, Estes P, Ramaswami M, Yamamoto D, Strausfeld NJ (1998) The organization of extrinsic neurons and their implications in the functional roles of the mushroom bodies in Drosophila melanogaster Meigen. Learn Mem 5:52-77. CrossRef Medline

Jan YN, Jan LY (2010) Branching out: mechanisms of dendritic arborization. Nat Rev Neurosci 11:316-328. CrossRef Medline

Jefferis GS, Marin EC, Stocker RF, Luo L (2001) Target neuron prespecification in the olfactory map of Drosophila. Nature 414:204-208. CrossRef Medline

Jefferis GS, Vyas RM, Berdnik D, Ramaekers A, Stocker RF, Tanaka NK, Ito K, Luo L (2004) Developmental origin of wiring specificity in the olfactory system of Drosophila. Development 131:117-130. CrossRef Medline

Kaufmann WE, Moser HW (2000) Dendritic anomalies in disorders associated with mental retardation. Cereb Cortex 10:981-991. CrossRef Medline

Komiyama T, Luo L (2006) Development of wiring specificity in the olfactory system. Curr Opin Neurobiol 16:67-73. CrossRef Medline

Komiyama T, Luo L (2007) Intrinsic control of precise dendritic targeting by an ensemble of transcription factors. Curr Biol 17:278-285. CrossRef Medline

Komiyama T, Johnson WA, Luo L, Jefferis GS (2003) From lineage to wiring specificity: POU domain transcription factors control precise connections of Drosophila olfactory projection neurons. Cell 112:157-167. CrossRef Medline

Komiyama T, Sweeney LB, Schuldiner O, Garcia KC, Luo L (2007) Graded expression of semaphorin-1a cell-autonomously directs dendritic targeting of olfactory projection neurons. Cell 128:399-410. CrossRef Medline

Lahaye LL, Wouda RR, de Jong AW, Fradkin LG, Noordermeer JN (2012) WNT5 interacts with the Ryk receptors doughnut and derailed to mediate muscle attachment site selection in Drosophila melanogaster. PLoS One 7:e32297. CrossRef Medline

Larsson MC, Domingos AI, Jones WD, Chiappe ME, Amrein H, Vosshall LB (2004) Or83b encodes a broadly expressed odorant receptor essential for Drosophila olfaction. Neuron 43:703-714. CrossRef Medline

Lee T, Luo L (1999) Mosaic analysis with a repressible neurotechnique cell marker for studies of gene function in neuronal morphogenesis. Neuron 22:451-461. CrossRef Medline

Mauss A, Tripodi M, Evers JF, Landgraf M (2009) Midline signalling systems direct the formation of a neural map by dendritic targeting in the Drosophila motor system. PLoS Biol 7:e1000200. CrossRef Medline

McAllister AK (2000) Cellular and molecular mechanisms of dendrite growth. Cereb Cortex 10:963-973. CrossRef Medline
Moreau-Fauvarque C, Taillebourg E, Boissoneau E, Mesnard J, Dura JM (1998) The receptor tyrosine kinase gene linotte is required for neuronal pathway selection in the Drosophila mushroom bodies. Mech Dev 78:4761. CrossRef Medline

Petrova IM, Malessy MJ, Verhaagen J, Fradkin LG, Noordermeer JN (2014) Wnt signaling through the ror receptor in the nervous system. Mol Neurobiol 49:303-315. CrossRef Medline

Rong YS, Golic KG (2000) Gene targeting by homologous recombination in Drosophila. Science 288:2013-2018. CrossRef Medline

Rosso SB, Inestrosa NC (2013) WNT signaling in neuronal maturation and synaptogenesis. Front Cell Neurosci 7:103. CrossRef Medline

Sakurai M, Aoki T, Yoshikawa S, Santschi LA, Saito H, Endo K, Ishikawa K, Kimura K, Ito K, Thomas JB, Hama C (2009) Differentially expressed Drl and Drl-2 play opposing roles in Wnt5 signaling during Drosophila olfactory system development. J Neurosci 29:4972-4980. CrossRef Medline

Schulte G, Bryja V (2007) The Frizzled family of unconventional G-proteincoupled receptors. Trends Pharmacol Sci 28:518-525. CrossRef Medline

Sharma Y, Cheung U, Larsen EW, Eberl DF (2002) PPTGAL, a convenient Gal4 P-element vector for testing expression of enhancer fragments in drosophila. Genesis 34:115-118. CrossRef Medline

Sweeney LB, Chou YH, Wu Z, Joo W, Komiyama T, Potter CJ, Kolodkin AL, Garcia KC, Luo L (2011) Secreted semaphorins from degenerating larval ORN axons direct adult projection neuron dendrite targeting. Neuron 72:734-747. CrossRef Medline

Tomlinson A, Struhl G (1999) Decoding vectorial information from a gradient: sequential roles of the receptors Frizzled and Notch in establishing planar polarity in the Drosophila eye. Development 126:5725-5738. Medline

Wagh DA, Rasse TM, Asan E, Hofbauer A, Schwenkert I, Dürrbeck H, Buchner S, Dabauvalle MC, Schmidt M, Qin G, Wichmann C, Kittel R, Sigrist SJ, Buchner E (2006) Bruchpilot, a protein with homology to ELKS/ CAST, is required for structural integrity and function of synaptic active zones in Drosophila. Neuron 49:833-844. CrossRef Medline

Walsh CA, Morrow EM, Rubenstein JL (2008) Autism and brain development. Cell 135:396-400. CrossRef Medline

Wong RO, Ghosh A (2002) Activity-dependent regulation of dendritic growth and patterning. Nat Rev Neurosci 3:803-812. CrossRef Medline

Yao Y, Wu Y, Yin C, Ozawa R, Aigaki T, Wouda RR, Noordermeer JN, Fradkin LG, Hing H (2007) Antagonistic roles of Wnt5 and the Drl receptor in patterning the Drosophila antennal lobe. Nat Neurosci 10: 1423-1432. CrossRef Medline

Yoshikawa S, McKinnon RD, Kokel M, Thomas JB (2003) Wnt-mediated axon guidance via the Drosophila Derailed receptor. Nature 422:583-588. CrossRef Medline

Zhang D, Zhou W, Yin C, Chen W, Ozawa R, Ang LH, Anandan L, Aigaki T, Hing H (2006) Misexpression screen for genes altering the olfactory map in Drosophila. Genesis 44:189-201. CrossRef Medline

Zhu H, Luo L (2004) Diverse functions of N-cadherin in dendritic and axonal terminal arborization of olfactory projection neurons. Neuron 42: 63-75. CrossRef Medline 